\title{
Lower rotational inertia and larger leg muscles indicate more rapid turns in tyrannosaurids than in other large theropods
}

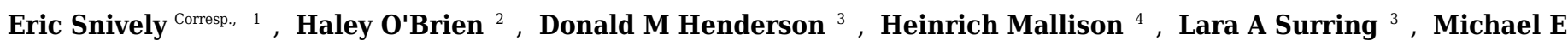
Burns $^{5}$ ， Thomas R Holtz, Jr. ${ }^{6,7}$ ， Anthony P Russell ${ }^{8}$, Lawrence M Witmer ${ }^{9}$ ， Philip J Currie ${ }^{10}$, Scott A Hartman ${ }^{11}$, John R Cotton ${ }^{12}$

${ }^{1}$ Department of Biology, University of Wisconsin-La Crosse, La Crosse, Wisconsin, United States

2 Department of Anatomy and Cell Biology, Oklahoma State University College of Osteopathic Medicine, Tulsa, Oklahoma, United States

3 Royal Tyrrell Museum of Palaeontology, Drumheller, Alberta, Canada

${ }^{4}$ Museum fur Naturkunde, Berlin, Germany

5 Department of Biology, Jacksonville State University, Jacksonville, Alabama, United States

6 Department of Geology, University of Maryland, College Park, Maryland, United States

7 Department of Paleobiology, National Museum of Natural History, Washington, D.C., United States

8 Department of Biological Sciences, University of Calgary, Calgary, Alberta, Canada

9 Department of Biomedical Sciences, Ohio University, Athens, Ohio, United States

10 Department of Biological Sciences, University of Alberta, Edmonton, Albeta, Canada

11 Department of Geoscience, University of Wisconsin-Madison, Madison, WI, United States

12 Department of Mechanical Engineering, Ohio University, Athens, Ohio, United States

Corresponding Author: Eric Snively

Email address: esnively@uwlax.edu

Synopsis: Tyrannosaurid dinosaurs had large preserved leg muscle attachments and low rotational inertia relative to their body mass, indicating that they could turn more quickly than other large theropods. Methods: To compare turning capability in theropods, we regressed agility estimates against body mass, incorporating superellipse-based modeled mass, centers of mass, and rotational inertia (mass moment of inertia). Muscle force relative to body mass is a direct correlate of agility in humans, and torque gives potential angular acceleration. Agility scores therefore include rotational inertia values divided by proxies for (1) muscle force (ilium area and estimates of $\mathrm{m}$. caudofemoralis longus crosssection), and (2) musculoskeletal torque. Phylogenetic ANCOVA (phylANCOVA) allow assessment of differences in agility between tyrannosaurids and non-tyrannosaurid theropods (accounting for both ontogeny and phylogeny). We applied conditional error probabilities $a(p)$ to stringently test the null hypothesis of equal agility. Results:

Tyrannosaurids consistently have agility index magnitudes twice those of allosauroids and some other theropods of equivalent mass, turning the body with both legs planted or pivoting over a stance leg. PhylANCOVA demonstrates definitively greater agilities in tyrannosaurids, and phylogeny explains nearly all covariance. Mass property results are consistent with those of other studies based on skeletal mounts, and between different figure-based methods (our main mathematical slicing procedures, lofted 3D computer 
models, and simplified graphical double integration). Implications: The capacity for relatively rapid turns in tyrannosaurids is ecologically intriguing in light of their monopolization of large $(>400 \mathrm{~kg}$ ), toothed dinosaurian predator niches in their habitats. 
1 Title

2

3 Lower rotational inertia and larger leg muscles indicate more rapid turns in

4 tyrannosaurids than in other large theropods

\section{Authors}

6 Eric Snively ${ }^{1}$, Haley O’Brien ${ }^{2}$, Donald M. Henderson ${ }^{3}$, Heinrich Mallison ${ }^{4}$, Lara A. Surring ${ }^{3}$,

7 Michael E. Burns ${ }^{5}$, Thomas R. Holtz Jr. ${ }^{6.7}$, Anthony P. Russell ${ }^{8}$, Lawrence M. Witmer ${ }^{9}$, Philip J.

8 Currie $^{10}$, Scott A. Hartman ${ }^{11}$, John R. Cotton ${ }^{12}$

\section{Affiliations}

$10{ }^{1}$ Deptartment of Biology, University of Wisconsin-La Crosse, La Crosse, WI, USA

$11{ }^{2}$ Department of Anatomy and Cell Biology, Oklahoma State University, Tulsa, OK, USA

$12{ }^{3}$ Royal Tyrrell Museum of Palaeontology, Drumheller, AB, Canada

$13 \quad{ }^{4}$ Museum für Naturkunde Berlin, Berlin, Germany

$14{ }^{5}$ Department of Biology, Jacksonville State University, Jacksonville, AB, USA

$15{ }^{6}$ Department of Geology, University of Maryland, College Park, MD, USA

$16{ }^{7}$ Department of Paleobiology, National Museum of Natural History, Washington, DC, USA

$17{ }^{8}$ Department of Biological Sciences, University of Calgary, Calgary, AB, Canada

$18{ }^{9}$ Department of Biomedical Sciences, Ohio University, Athens, OH, USA

$19{ }^{10}$ Department of Biological Sciences, University of Alberta, Edmonton, AB, Canada

$20{ }^{11}$ Department of Geoscience, University of Wisconsin, Madison, WI, USA

$21{ }^{12}$ Department of Mechanical Engineering, Russ College of Engineering and Technology, Ohio

22 University, Athens, OH, USA

\section{Corresponding Author}

Eric Snively

Dept. of Biology

University of Wisconsin-La Crosse

1725 State Street

La Crosse, WI 54601 


\section{Abstract}

35

36

Synopsis: Tyrannosaurid dinosaurs had large preserved leg muscle attachments and low rotational inertia relative to their body mass, indicating that they could turn more quickly than other large theropods. Methods: To compare turning capability in theropods, we regressed agility estimates against body mass, incorporating superellipse-based modeled mass, centers of mass, and rotational inertia (mass moment of inertia). Muscle force relative to body mass is a direct correlate of agility in humans, and torque gives potential angular acceleration. Agility scores therefore include rotational inertia values divided by proxies for (1) muscle force (ilium area and estimates of m. caudofemoralis longus cross-section), and (2) musculoskeletal torque. Phylogenetic ANCOVA (phylANCOVA) allow assessment of differences in agility between tyrannosaurids and non-tyrannosaurid theropods (accounting for both ontogeny and phylogeny). We applied conditional error probabilities $\alpha(p)$ to stringently test the null hypothesis of equal agility. Results: Tyrannosaurids consistently have agility index magnitudes twice those of allosauroids and some other theropods of equivalent mass, turning the body with both legs planted or pivoting over a stance leg. PhylANCOVA demonstrates definitively greater agilities in tyrannosaurids, and phylogeny explains nearly all covariance. Mass property results are consistent with those of other studies based on skeletal mounts, and between different figurebased methods (our main mathematical slicing procedures, lofted 3D computer models, and simplified graphical double integration). Implications: The capacity for relatively rapid turns in tyrannosaurids is ecologically intriguing in light of their monopolization of large $(>400 \mathrm{~kg})$, toothed dinosaurian predator niches in their habitats. 


\section{Introduction}

58

Tyrannosaurid theropods were ecologically unusual dinosaurs (Brusatte et al. 2010), and were as adults the only toothed terrestrial carnivores larger than $60 \mathrm{~kg}$ (Farlow and Holtz 2002) across much of the northern continents in the late Cretaceous. They ranged in adult trophic morphology from slender-snouted animals such as Qianzhousaurus sinensis (Li et al. 2009, Lü et al. 2014) to giant bone-crushers including Tyrannosaurus rex (Rayfield 2004, Hurum and Sabath 2003, Snively et al. 2006, Brusatte et al. 2010, Hone et al. 2011, Bates and Falkingham 2012, Gignac and Erickson 2017). In addition to the derived features of their feeding apparatus, the arctometatarsalian foot of tyrannosaurids likely contributed to effective prey capture through rapid linear locomotion and enhanced capability of the foot to resist torsion when maneuvering (Holtz 1995, Snively and Russell 2003, Surring et al., in revision). Features suggestive of enhanced agility (rate of turn) and tight maneuverability (radius of turn) in tyrannosaurids include relatively short bodies from nose to tail (anteroposteriorly short thoracic regions, and cervical vertebrae that aligned into posterodorsally retracted necks), small forelimbs, and long, tall ilia for leg muscle attachment (Paul 1988, Henderson and Snively 2003, Bakker and Bir 2004, Hutchinson et al. 2011). Here we present a biomechanical model that suggests tyrannosaurids could turn with greater agility, thus pivoting more quickly, than other large theropods, suggesting enhanced ability to pursue and subdue prey.

Like other terrestrial animals, large theropods would turn by applying torques (cross products of muscle forces and moment arms) to impart angular acceleration to their bodies. This angular acceleration can be calculated as musculoskeletal torque divided by the body's mass moment of inertia (=rotational inertia). Terrestrial vertebrates such as cheetahs can induce a tight turn by lateroflexing and twisting one part of their axial skeleton, such as the tail, and then 
80 rapidly counterbending with the remainder, which pivots and tilts the body (Wilson et al. 2013,

81 Patel and Braae 2014, Patel et al. 2016). The limbs can then accelerate the body in a new

82 direction (Wilson et al. 2013). These tetrapods can also cause a larger-radius turn by accelerating

83 the body more quickly with one leg than the other (pushing off with more force on the outside of

84 a turn), which can incorporate hip and knee extensor muscles originating from the ilium and tail

85 (Table 1). Hence muscles originating from the ilium can cause yaw (lateral pivoting) of the entire

86 body, although they do not induce yaw directly. Such turning balances magnitudes of velocity

87 and lean angle, and centripetal and centrifugal limb-ground forces. When limbs are planted on

88 the ground, the body can pivot with locomotor muscle alone. In either case, limb muscles actuate

89 and stabilize their joints, positively accelerating and braking the body and limbs.

$90 \quad$ Forces from locomotor muscles have a fundamental influence on agility. Torques from

91 these limb muscles are necessary for estimating absolute angular acceleration (Hutchinson et al.

92 2007), and muscle power also influences turning rate (Young et al. 2002). Experimental trials

93 with human athletes show that agility scales with muscle force production relative to body mass

94 (Peterson et al. 2006). Measures involving mass-specific force production, such as plyometric

95 performance, correlate positively with agility tests of linear braking and accelerating (Peterson et

96 al. 2006) and trials involving angular acceleration (Thomas et al. 2009, Markovic 2007,

97 Anderson et al. 1991). Eccentric force, particularly of hamstrings in humans, is especially

98 important for angular changes of direction (Anderson et al. 1991). Isolated indicators of maximal

99 force of knee extensors alone contribute no more than $20 \%$ of variance to agility tests (Markovic

100 2007), whereas functional, integrated force production and neurological training have larger

101 effects on agility (Peterson et al. 2006, Markovic et al. 2007). 

to investigate relative agility. Muscle force is proportional to physiological cross sectional area

104 (PCSA), and in turn on muscle volume, pennation angle, and dramatically on fiber length (Bates and Falkingham 2018), in addition to maximal isometric stress and activation level. Muscle anatomical cross sectional area and hence volume vary proportionally with attachment size of

107 homologous muscles (explained in detail under Methods). In fossil taxa, attachment size is a consistent, reliably preserved influence on muscle force. Relative muscle force is therefore a useful, replicable metric for comparative assessments of agility in fossil tetrapods. Estimates of

110 theropod muscle force and the mass properties of their bodies can facilitate comparisons of

111 turning ability in theropods of similar body mass.

112 This relative agility in theropods is testable by regressing estimated body mass (Fig. 1)

113 against indicators of agility, which incorporate fossil-based estimates of muscle force (Fig. 2),

114 torque, and body mass and mass moment of inertia $\left(I_{y} ;\right.$ Fig. 1$)$. Given the same moment arm

115 lengths, greater force relative to rotational inertia indicates the ability to turn more rapidly.

116 Coupled with protracted juvenile growth periods (Erickson et al. 2004), heightened agility would

117 be consistent with the hypothesis that tyrannosaurids were predominantly predatory, and help to

118 explain how late Campanian and Maastrichtian tyrannosaurids monopolized the large predator

119 niche in the Northern Hemisphere.

120 Estimating mass properties and comparative turning performance of carnivorous dinosaurs

121 To compare agility in theropods, we divided ilium area (a proxy for muscle cross

122 sectional area and maximal force production), and estimated $\mathrm{m}$. caudofemoralis longus cross-

123 sections, by $I_{y}$ (rotational inertia in yaw about the body's center of mass). We also incorporated

124 scaling of moment arm size in a separate analysis to better compare absolute turning 
125 performance in the theropods. We restrict our comparisons to proxies of agility at given body

126 masses, rather than estimating absolute performance, because a generalized predictive approach

127 enables us to compare many taxa. Viable paths for testing our results include musculoskeletal

128 dynamics of turning involving all hind limb muscles, as undertaken by Rankin et al. (2016) for

129 linear locomotion in ostriches, or simpler approaches such as Hutchinson et al.'s (2007)

130 calculations for turning in Tyrannosaurus. However, the dynamics of turning are complicated to

131 pursue even in extant dinosaurs (Jindrich et al. 2007), and estimating absolute performance in

132 multiple extinct taxa would entail escalating numbers of assumptions with minimal comparative

133 return. We therefore focus here on relative metrics of turning performance, based as much as

134 possible on direct fossil data.

135 Using relative indices of agility, encompassing origins for relevant ilium-based muscles,

136 tail-originating muscles (Table 1), and mass moments of inertia, enables us to address action

137 beyond yaw alone. Muscles of the leg on the outside of a turn normally involved in linear

138 motion would change the body's direction by linearly accelerating the body in that direction,

139 while muscles for the leg on the inside of the turn exert less torque. Muscles involved in

140 stabilizing the limbs and body, and providing contralateral braking and abduction, would come

141 into play during rotation of the body (Anderson et al. 1991). Mass moment of inertia is the most

142 stringent mass-property limit on turning ability in long, massive dinosaurs (Carrier et al. 2001,

143 Henderson and Snively 2003). This simplified approach is predictive, testable with more

144 complex investigations (including specific torques of muscle-bone couples: Hutchinson et al.

145 2007), and allows broad comparisons of overall turning ability.

146 Our hypotheses of comparative agility in large theropods incorporate two behavioral

147 scenarios potentially important for prey capture. 
148 Hypothesis 1: Tyrannosaurids could turn their bodies more quickly than other theropods when

149 close to prey, pivoting the body with both feet planted on the ground.

150 Hypothesis 2. Tyrannosaurids could turn more quickly than other theropods when approaching

151 prey, pivoting the body plus a suspended swing leg above one stance foot planted on the ground.

152 Under the scenario in Hypothesis 1, the applicable mass moment of inertia $I_{y}$ is that of the

153 body not including the hind legs, about a vertical axis through the body's center of mass.

154 Intuitively the body would yaw about a vertical line between the acetabula, but the centers of

155 mass of bipedal dinosaurs, and therefore their feet and ground reaction forces in this stance, are

156 almost always estimated to be anterior to the acetabulum (Henderson 1999, Hutchinson et al.

157 2007, Allen et al. 2009, Bates et al. 2009a, b; Hutchinson et al. 2011, Bates et al. 2012, Allen et 158 al. 2013).

159 In a prey pursuit scenario under Hypothesis 2, the theropod has just pushed off with its

160 swing leg, and is pivoting about its stance leg as it protracts the swing leg. The body and swing

161 leg are rotating about their collective center of mass (COM), directly above the stance foot. Total

$162 I_{y}$ in this case includes the entire axial body (minus the hind legs), and the contribution of the

163 swing leg to total $I_{y}$ of the system.

165 Materials and methods

166 Comparing relative turning performance in tyrannosaurids and other theropods requires

167 data on mass moment of inertia $I_{y}$ about a vertical axis (y) through the body's center of mass

168 (COM), and estimates of leg muscle force and moment arms. ( $I$ in this paper always refers to

169 mass moment of inertia, not $I$ as the common variable for area moment of inertia.) To estimate

170 mass, COM, and $I_{y}$, we approximated the bodies of the theropods as connected frusta (truncated 
171 cones or pyramids) with superellipse cross-sections (Fig. 1). Superellipses are symmetrical

172 shapes the outline of which (from star-shaped, to ellipse, to rounded rectangle) are governed by

173 exponents and major and minor dimensions (Rosin 2000, Motani 2001, Snively et al. 2013).

174 Spreadsheet templates for calculations of dimensions, mass, centers of mass, and

175 rotational inertias are available as supplementary information. These enable the estimation of

176 mass properties from cross-sectional and length dimensions, using Microsoft Excel-compatible

177 software. Snively et al. (2013) provide coefficients and polynomial regression equations for

178 super-elliptical frusta.

179 Specimens

180 Theropod specimens (Table 2) were included if they had complete ilia, and relatively

181 complete skeletons ideally including the tail. If tails were incomplete they were reconstructed

182 from other specimens of the same or a closely related genus, following the practice of Taylor

183 (2009). Tyrannosaurid adults and juveniles are well represented by complete skeletons. Most

184 other taxa were allosauroids, many of which are known from complete or rigorously

185 reconstructable skeletons. Yangchuanosaurus shangyouensis and Sinraptor hepingensis are basal

186 allosauroids. Their relative Sinraptor dongi lacks a preserved tail, and the older

187 Monolophosaurus jiangi has a complete axial skeleton but lacks preserved hind legs, which are

188 necessary for reliable mass estimates. Both species were therefore omitted. An early relative of

189 allosauroids and tyrannosaurs, Eustreptospondylus oxoniensis, was included as a nearly complete,

190 small representative of an allosauroid body plan, because it has a similar ratio of ilium/femur

191 length as a less-complete juvenile specimen of Allosaurus fragilis (Foster and Chure 2006), and

192 is a reasonable proxy for the basal allosauroid condition. The non-tetanuran theropods

193 Dilophosaurus wetherelli and Ceratosaurus nasicornis were included for their similarity in size 
194 to juvenile tyrannsaurids, and to enable examination of how phylogeny affects patterns of mass

195 moment of inertia versus muscle force. We include the small tyrannosaur that Sereno et al. (2009)

196 named Raptorex kriegsteini. Fowler et al. (2011) provide evidence that this specimen is a

197 juvenile Tarbosaurus bataar (see also Brusatte and Carr 2016). We informally refer to it as

198 Raptorex to differentiate it from a much larger juvenile Tarbosaurus in our sample.

199 Digitizing of body outlines

200 Technical skeletal reconstructions by Paul $(1988,2010)$ and Hartman $(2011)$, in dorsal

201 and lateral views, were scanned on a flatbed scanner or saved as images (Hartman 2011),

202 vectorized with the Trace function in Adobe illustrator, and "expanded" for editing the entire

203 outlines and individual bones. Lateral and dorsal outlines were modified based on body

204 dimensions such as trunk, neck, and head length, and trunk and tail depth, as measured from

205 scaled figures in the primary literature (Osborn 1917; Gilmore 1920; Russell 1970; Dong 1983;

206 Gao 1992; Brochu 2003; Bates 2009a, b) and photographs of skeletons. We modified these

207 outlines with updated anatomical data on neck and tail dimensions (Snively and Russell 2007a,

208 Allen et al. 2009, Persons and Currie 2010), and the jaws were positioned as closed. The

209 chevrons of Giganotosaurus were angled posteroventrally to match those of its relatives

210 Acrocanthosaurus and Allosaurus. Dorsal and lateral views were scaled to the same length, and

211 divided into 60+ segments with lines crossing corresponding structures in both views (Fig. 1).

212 Coordinates were digitized for dorsal, ventral, midsagittal, and lateral contours using

213 PlotDigitizer (Huwaldt 2010), scaled to femur lengths of the specimens. Coordinates were

214 opened as CSV data in Microsoft Excel.

215 If a dorsal reconstruction of the skeleton was unavailable, a dorsal view of the animal's

216 nearest relative was modified (Taylor 2009). Ideally this relative is the immediate sister taxon or 
217 another specimen of the same species but at a different growth stage (as with young

218 Gorgosaurus and Tyrannosaurus). Anterior and posterior extremes of the head, neck, trunk

219 (coracoids to anterior edge of ilium), ilium, and tail were marked on the lateral view. The

220 corresponding structures on the dorsal view were selected and modified to match their

221 anteroposterior dimensions in the lateral view. Width of the surrogate dorsal view was modified

222 based on literature- or specimen-based width measurements of available structures. For example,

223 many transverse measurements of a juvenile Tyrannosaurus rex skeleton (BMR P2002.4.1;

224 courtesy of Scott Williams) were used to modify a dorsal view of an adult (Persons and Currie

225 2011a). The distal portion of the tail in Yangchuanosaurus was modeled on the more complete

226 tail of Sinraptor hepingensis.

227 If a dorsal view of only the skull was available for a given dinosaur, and a dorsal view of

228 the skeleton was only available for a related taxon, the differential in skull widths between the

229 taxa was applied to the entire dorsal view of the relative's skeleton. When possible we used

230 transverse widths of occipital condyles and frontals, measured by author PJC, to confirm ratios

231 of total reconstructed skull widths. The width of the occipital condyle reflects width of the atlas

232 and postaxial cervical vertebrae, and hence influences width of remaining vertebrae as well. This

233 wholesale modification of body width is therefore tentative, but uses the best-constrained

234 available data, and is testable with future, more complete descriptions and measurements of

235 theropod postcrania. We applied this method for dorsal reconstructions of Sinraptor,

236 Eustreptospondylus, Dilophosaurus, Tarbosaurus, and one juvenile Gorgosaurus. For example,

237 for Eustreptospondylus the skull width from Walker (1964) was used to modify a dorsal

238 reconstruction of Allosaurus, and the skull width of Sinraptor hepingensis was applied to a

239 dorsal view of its close relative Yangchuanosaurus shangyouensis. Ribcage width in individual 
240 animals varies with ventilatory movements, but width variations of $+/-10 \%$ (Henderson and

241 Snively 2003, Bates et al. 2009) have sufficiently small effect on $I_{Y}$ to permit statistically valid

242 comparisons (see Henderson and Snively 2003).

243 We also digitized the hind legs of the specimens, by extending their skeletons and soft

244 tissue outlines to obtain anterior and posterior coordinates. We applied a uniform semi-minor

245 axis in the mediolateral direction, as a radius from the midline of the femur to the lateral extent

246 of its reconstructed musculature (Paul 1988, 2010). The anterior and posterior points on the ilium

247 constrained the maximum anteroposterior extent of the thigh muscles (Hutchinson et al. 2005),

248 which we tapered to their insertions at the knee. The anterior point of the cnemial crest

249 constrained the anterior extent of the crural muscles, but the posterior contours were admittedly

250 subjective. In Paul's $(1988,2010)$ reconstructions, the posterior extent of the $\mathrm{m}$. gasctrocnemius

251 complex in lateral view (bulge of the "drumstick" muscles) generally correlates with the width of

252 the distal portion of the femoral shaft, where two bellies of these muscles originate. Masses of

253 both legs were added to that of the axial body to obtain total body mass. Forelimbs were not

254 included, because they could not be digitized for all specimens and add proportionally little to

255 overall mass moments of inertia (Henderson and Snively 2003, Bates et al. 2009a). The reduced

256 forelimbs of tyrannosaurids would likely add less to overall body $\mathrm{I}_{\mathrm{Y}}$ than the larger forelimbs of

257 other large theropods, especially with shorter glenoacetabular distance in tyrannosaurids (Paul

258 1988). However, even the robust forelimbs of Acrocanthosaurus, for example, would contribute

259 only $0.15 \%$ of the $I_{y}$ of its entire axial body (Bates et al. 2009a).

260 Mass property estimates

261 Volume and mass 
Body volume, mass, center of mass (COM), and mass moment of inertia were calculated

263

264

265

266

267

268

269

270

271

272

273

274

275

276

277

278

279

280

281

282

283

284

using methods similar to those of Henderson (1999), Motani (2001), Henderson and Snively

(2003), Durkin and Dowling (2006), and Arbour (2009). Body segments were approximated as

frusta (truncated cones), and volume of the axial body calculated as the sum of volumes of

constituent frusta (mass estimates incorporated regional densities of the body; see below).

Coordinates for midsagittal and coronal outlines were used to calculate radii for anterior and posterior areas of each frustum. Arbour (2009) thoroughly explains the equations and procedures for calculating volume of conical frusta. Equation 1 is for volume of an elliptical frustum, in notation of radii (r) and length (1).

$$
\text { 1) } \quad V=\frac{\pi}{3} \times l\left(r_{\text {ant }}^{D V} r_{\text {ant }}^{L M}+r_{\text {post }}^{D V} r_{\text {post }}^{L M}+\sqrt{r_{\text {ant }}^{D V} r_{\text {ant }}^{L M} r_{\text {post }}^{D V} r_{\text {post }}^{L M}}\right)
$$

The superscript $D V$ refers to a dorsoventral radius, and $L M$ the lateral-to-midsagittal dimension (Fig. 2).

This equation can be generalized to frustum face areas of any cross section (equation 2; similar to equations presented by Motani [2001] and Arbour [2009]).

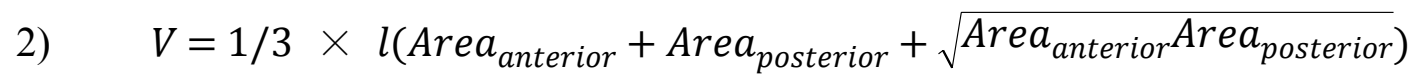

Using equation 2, frustum volumes can be calculated from cross sections departing from that of an ellipse. Vertebrate bodies deviate from purely elliptical transverse sections (Motani 2001). We therefore calculated areas based on a range of superellipse exponents, from 2 (that of an ellipse) to 3 (as seen in whales and dolphins), based on the derivations and correction factors of Snively 
285 (2012) and Snively et al. (2013). Exponents for terrestrial vertebrates range from 2-2.5, with 2.5

286 being common (Motani 2001; Snively and Russell [2007b] used 2.3). Snively (2012) and Snively

287 et al. (2013) derived and mathematically validated constants for other superelliptical cross-

288 sections; for example, for $k=[2,2.3,2.4,2.5], C=[0.7854,0.8227,0.8324,0.8408]$. Volumes for

289 different cross sections were then calculated by applying these constants, as superellipse

290 correction factors (Snively et al. 2013), to equations 1 and 2.

291 Frustum volumes were multiplied by densities to obtain masses, and these were summed

292 to obtain axial-body and leg masses. For the head we applied average density of $990 \mathrm{~kg} / \mathrm{m}^{3}$,

293 based on an exacting reconstruction of bone and air spaces in Allosaurus by Snively at al. (2013).

294 We used a neck density of $930 \mathrm{~kg} / \mathrm{m}^{3}$ and trunk density of $740 \mathrm{~kg} / \mathrm{m}^{3}$ similar to that of Bates et al.

295 (2009) for the same specimen of Allosaurus, which also accounted for air spaces. The post-

296 thoracic and leg densities were set to that of muscle at $1060 \mathrm{~kg} / \mathrm{m}^{3}$. Density and resulting mass of

297 these anatomical regions was probably greater (even if fat is included) because bone is denser

298 than muscle, which would result in a more posterior COM than calculated here. Rather than

299 introduce new sets of assumptions, we provisionally chose muscle density because its value is

300 known, and the legs (Hutchinson et al. 2011) and tail (Mallison et al. 2015) have far greater

301 volumes of muscle than bone. All of these density values are easily modifiable in the future, as

302 refined anatomical data for air spaces, bone densities, and bone volumes become available, such

303 as occurred with the restoration methods of Witmer and Ridgely (2008) and Snively et al. (2013).

We also varied tail cross-sections by applying the results of Mallison et al. (2015) for the

m. caudofemoralis longus and full-tail cross sections of adult Alligator mississippiensis and other

307 crocodilians. Mallison et al. (2015) found that proximal cross-sections of an adult Alligator tail 
308 and $\mathrm{m}$. caudofemoralis longus are 1.4 times greater than those previously estimated for young

309 Alligator and dinosaurs (Persons and Currie (2011a). We therefore multiplied the original width

310 of the modeled tails of theropods (see above) by 1.4 to obtain an upper estimate of tail thickness

311 and mass.

312 Inter-experimenter variation in reconstruction

313 We checked our mass estimation method against that of Bates et al. (2009a) by digitizing

314 their illustrations of Acrocanthosaurus atokensis, including the body and the animal's dorsal fin

315 separately. The dorsal fin was restored with half a centimeter of tissue on either side the neural

316 spines, with a bony width of approximately $4 \mathrm{~cm}$ that Harris (1998) reported for the twelfth

317 dorsal vertebra. We assumed a rectangular cross section for the fin. The digitization and mass

318 property estimates (see below) for Acrocanthosaurus were purposely carried out blind to the

319 results of Bates et al. (2009a), to avoid bias in scaling and digitizing the outline of their

320 illustrations.

$321 \quad$ Authors DMH and ES independently digitized reconstructions and estimated mass

322 properties of several specimens, including the legs of many specimens and axial bodies of

323 Ceratosaurus, Allosaurus, adult Gorgosaurus, and Daspletosaurus. The software and coding

324 differed in these attempts, and volume reconstruction equations differed slightly (Henderson

325 1999, Snively 2012; current paper). To further evaluate inter-experimeter variation in results, ES

326 and graduate student Andre Rowe separately used the current paper's methods to digitize an adult

327 Gorgosaurus. (In all cases discrepancies were negligible, and we were confident to proceed; see

328 Results.)

329 Centers of mass 
331 mass (COM) of the axial bodies (not including the legs), assuming that the animal would pivot

332 the body around this location if both legs were planted on the ground. First, we calculated the

333 center of mass of each frustum. Equation 3 gives the anteroposterior position of each frustum's

$334 \operatorname{COM}\left(C O M_{A P}\right) ; r$ are radii of anterior and posterior frusta, and $L$ is its length (usually designated

335 "h" for height of a vertical frustum).

336

3) $\quad C O M_{\text {frustum } A P}=\frac{L \times\left(r_{\text {ant }}^{2}+2 r_{\text {ant }} r_{\text {post }}+3 r_{\text {post }}^{2}\right)}{4 \times\left(r_{\text {ant }}^{2}+r_{\text {ant }} r_{\text {post }}+r_{\text {post }}^{2}\right)}$

337 Equation 4 below is an approximation of the dorsoventral position of a frustum's center of mass

$338\left(C O M_{\text {frustum } D V}\right)$, from digitized y (height) coordinates of the lateral body outlines. In this equation,

$339 h_{\text {ant }}$ and $h_{\text {post }}$ are the full heights (dorsoventral dimensions) of the anterior and posterior faces of

340 the frustum, equal to twice the radii $r$ in equation 3. The absolute value terms (first and third in

341 the numerator) ensure that the result is independent of whether or not the anterior or posterior

342 face is taller.

343

4) $\quad C O M_{\text {frustum } D V}=\frac{2 \times h_{\text {ant }}\left|h_{\text {post }}-h_{\text {ant }}\right|+h_{\text {ant }}^{2}+h_{\text {post }}\left|h_{\text {post }}-h_{\text {ant }}\right|+h_{\text {ant }} h_{\text {post }}+h_{\text {post }}^{2}}{3 \times h_{\text {ant }}+h_{\text {post }}}$

345 Equation 4 gives an exact $C O M_{\text {frustum } D V}$, but assumes that all frustum bases are at the same height

346 (as though they are all resting on the same surface). To obtain the y (vertical) coordinate for the

347 COM of each animal's body, we first approximated $C O M_{\text {frustum } D V}$ using dorsal and ventral

348 coordinates of the anterior and posterior face of each frustum (equation 5).

349

$$
C O M_{\text {frustum } D V}=\frac{\left[\left(y_{\text {ant:dorsal }}+y_{\text {ant:venral }}\right)+\left(y_{\text {post:dorsal }}+y_{\text {post:venral }}\right)\right]}{4}
$$

We obtained the center of mass $C O M_{b o d y}$ for the entire axial body (both anteroposterior

351 and dorsoventral), by multiplying the mass of each frustum $i$ by its position, summing these 
352 quantities for all frusta, and dividing by the entire axial body mass (equation 6). This gives the

353 anteroposterior $C O M_{A P}$ from the tip of the animal's rostrum, and the dorsoventral $C O M_{D V}$ at the

354 depth of $C O M_{A P}$ above the ventral-most point on the animal's trunk (typically the pubic foot).

355

6) $\quad C O M_{\text {body }}=\frac{\sum_{i=1}^{n} \operatorname{COM}_{\text {frustum } i} \times m_{\text {frustum } i}}{m_{\text {body }}}$

To test Hypothesis 2, we found the position of collective COM of the body and leg,

$357 C O M_{b o d y+l e g}$, which lies lateral to $C O M_{b o d y}$ calculated in equation 6. The lateral (z) coordinate of $358 C O M_{b o d y-z}$ was set to 0 , and that of the leg $C O M_{\text {leg-z }}$ was measured as the distance from $C O M_{b o d y: z}$

359 to the centroid of the most dorsal frustum of the leg. Equation 7 enables calculation of

$360 C O M_{b o d y+l e g: z}$ with this distance $C O M_{\text {leg:z }}, C O M_{b o d y: z}$, and the masses of the swing leg and axial

361 body.

7) $\quad C O M_{\text {body }+ \text { leg:z }}=\frac{\operatorname{COM}_{\text {body:z }} m_{\text {body }}+\operatorname{COM}_{\text {leg: }} m_{\text {leg }}}{m_{\text {body }}+\text { leg }}$

363

364 Mass moments of inertia: Hypothesis 1 (both legs planted)

365 Mass moment of inertia for turning laterally, designated $I_{y}$, was calculated about the axial

366 body's COM by summing individual $I_{\mathrm{y}}$ for all frusta (equation 8 , first term), and the contribution

367 of each frustum to the total using the parallel axis theorem (equation 8 , second term).

368

8) $\quad I_{y}=\sum_{i=1}^{n}\left(\frac{\pi}{4}\right) \rho_{i} l_{i} \bar{r}_{D V} \bar{r}_{L M}^{3}+m_{i} r_{i}^{2}$

369 For calculating $I_{\mathrm{y}}$ of an individual frustum, $\rho_{i}$ is its density, and $l_{i}$ is its anteroposterior length.

370 The element $\pi / 4$ is a constant $(C)$ for an ellipse, with an exponent $k$ of 2 for its equation. We

371 modified $C$ with superellipse correction factors for other shapes (Snively et al. 2013). The

372 dimension $\bar{r}_{D V}$ is the average of dorsoventral radii of the anterior and posterior faces of each

373 frustum, and $\bar{r}_{L M}$ are the average of mediolateral radii. The mass $m_{i}$ and COM of each frustum 
374 were calculated using the methods described above, and distance $r_{i}$ from the whole body's COM

375 to that of each frustum was estimated by adding distances between each individual frustum's

376 COM to that of frustum $i$.

377 Mass moments of inertia: Hypothesis 2 (pivoting about the stance leg)

$378 \quad$ Here the body and leg are pivoting in yaw about a vertical axis passing through their

379 collective center of mass $C O M_{b o d y+l e g}$, and the center of pressure of the stance foot. Here

380 rotational inertia $I_{y \text { body }+ \text { leg }}$ about the stance leg is the sum of the four right terms in equation 9.

381

9) $\quad I_{y b o d y+l e g}=I_{y b o d y}+I_{y l e g}+m_{\text {body }} r_{C O M-t o-b o d y}+m_{\text {leg }} r_{\text {COM }-t o-l e g}^{2}$

382 Term 1. $I_{y \text { body }}$ of the axial body about its own COM;

383 Term 2. $I_{y \text { leg }}$ of the swing leg about its own COM (assuming the leg is straight);

384 Term 3. The axial body's mass $m_{\text {body }}$ multiplied by the square of the distance $r_{C O M-t o-b o d y}$ from its

385 COM to the collective COM of the body + swing leg $\left(C O M_{b o d y+l e g}\right)$;

386 Term 4 . The swing leg's mass $m_{\text {leg }}$ multiplied by the square of the distance $r_{\text {COM-to-leg }}$ from its

387 COM to the collective COM of the body + swing leg $\left(C O M_{b o d y+l e g}\right)$.

388 We calculated $I_{y \text { body }}$ using equation 8. To calculate $I_{y \text { leg }}$ (equation 10), we approximate

389 the swing leg as extended relatively straight and rotating on its own about an axis through the

390 centers of its constituent frusta. In equation $10, I_{y}$ leg is the sum of $I_{y \text { frustum }}$ for all individual frusta

391 of the leg, and $I_{y \text { frustum }}$ is in turn simply the sum of $I_{x}$ and $I_{z}$ of each frustum (Durkin 2003). These

392 are similar to the first term in equation 8 , but with anteroposterior radii $r_{A P}$ instead of the

393 dorsoventral radius of frusta of the axial body.

10) $\quad I_{y \text { leg }}=\sum_{i=1}^{n}\left(\frac{\pi}{4}\right) \rho_{i} l_{i}\left(\bar{r}_{A P} \bar{r}_{L M}^{3}+\bar{r}_{L M} \bar{r}_{A P}^{3}\right)$

395 Equations 11 and 12 give distance $r_{C O M-t o-b o d y}$ and $r_{C O M-t o-l e g}$ necessary for equation 9; note the

396 brackets designating absolute values, necessary to find a distance rather than a $\mathrm{z}$ coordinate. 
397 11) $\quad r_{C O M-t o-b o d y}=\left|C O M_{b o d y+l e g}-C O M_{b o d y}\right|$

398 12) $\quad r_{\text {COM - to-leg }}=\mid \operatorname{COM}_{\text {body }+ \text { leg }}-$ COM $_{\text {leg }} \mid$

399 An Excel spreadsheet in Supplementary Information (theropod_RI_body+one_leg.xlsx) has all

400 variables and equations for finding RI of the body plus leg.

401 Estimating areas of muscle origination and cross-section

402 We obtained proxies for muscle force by estimating areas of muscle attachment and 403 cross-section (Fig. 2). Muscle cross-section, and therefore force, scales at a gross level with 404 attachment area for homologous muscles between species, for example with the neck muscles of 405 lariform birds (Snively and Russell 2007a). Enthesis (attachment) size for individual muscles 406 does not scale predictably with force within mammalian species of small body size (Rabey et al.

407 2014, Williams-Hatala et al. 2016), which necessitates a more general proxy for attachment area 408 and force correlations between taxa, across spans of evolutionary time (Moen et al. 2016).

409 In such interspecific comparisons, morphometrics establish correlation between general

410 muscle origin size and locomotor ecomorphologies (Moen et al. 2013, 2016; Tinius et al. 2018).

411 Leg length and ilium size are associated with both muscle size and jumping performance in frogs,

412 across biogeography, phylogeny, and evolution (Moen et al. 2013, 2016). Between species of

413 Anolis lizards, the overall size of muscle attachments on the ilium correlates with necessities of

414 force and moments in different ecomorphotypes, including small and large ground dwellers, 415 trunk and branch climbers, and crown giants (Tinius et al. 2018).

416 In theropods, the ilium is the most consistently preserved element that records leg muscle

417 origination, and is usable for estimating overall origin area of knee extensors, hip flexors, and

418 femoral abductors (Table 2). In large theropods, these enthesis regions have similar gross 
419 morphology, including striations indicating Sharpey's fiber-rich origins for the divisions of the $\mathrm{m}$.

420 iliotibialis, and smooth surfaces for the m. iliofemoralis.

421 Because ilium attachment sites are similar in all theropods, as a reasonable first

422 approximation we infer greater forces for muscles originating from ilia with substantially greater

423 attachment areas than smaller ones (for example, twice as long and tall). Ilia of large theropod

424 species have a preacetabular flange with a ventral projection, which some authors reconstruct as

425 origin for an anterior head of m. iliotibialis. We include this region in area calculations, but the

426 flange is conceivably also or alternatively an origin for $\mathrm{m}$. iliocostalis, which would stabilize the

427 trunk.

428 We make similar assumptions for interspecies comparisons of the major femoral retractor,

429 the m. caudofemoralis longus (CFL). The depth of the tail ventral to the caudal ribs correlates

430 with the cross-section of the CFL (Persons and Currie 2011a,b; Hutchinson et al. 2011, Mallison

431 et al. 2015). Although complete tails are rarely preserved (Hone 2012), the depth of the proximal

432 portion of the tail permits a good first estimation of maximum CFL cross-section (Persons and

433 Currie 2011a, b; Mallison et al. 2015).

434 Another femoral retractor, the m. caudofemoralis brevis (CFB), originates from the brevis

435 fossa of the postacetabular region of the ilium. We chose to omit the area of origin of the CFB

436 from this analysis, because this would require a ventral view of the ilium, which is rarely figured

437 in the literature and is difficult to photograph on mounted skeletons. A dorsal view might suffice

438 as a proxy for width of the brevis fossa, but the fossa is flanked by curved alae of bone whose

439 width is obscured in dorsal view. The fossa, and presumably the origination attachment for the

440 CFB (Carrano and Hutchinson 2002), is longer in tyrannosaurids than in other theropods because 
441 the ilia are longer relative to body length (Paul 1988), but not broader (Carrano and Hutchinson

442 2002; figures in Osborn 1917, Gilmore 1920, and Madsen 1976).

443 Ilium area for muscle attachment was determined for all taxa from lateral-view

444 photographs and scientific illustrations (Table 2) scaled to the size of the original specimen (Fig.

445 2). Because some muscle scars are ambiguous, the entire lateral surface of the ilium dorsal to the

446 supra-acetabular crest was considered as providing potential area for muscle origination. Images

447 were opened in ImageJ (United States National Institutes of Health, Bethesda, Maryland, USA),

448 scaled in $\mathrm{cm}$ to the size of the original specimens, and the bone areas outlined. ImageJ (under

449 "Measure") was used to calculate areas within the outlines in $\mathrm{cm}^{2}$.

450 Relative cross-sections were reconstructed for the m. caudofemoralis longus (CFL),

451 although the sample size is smaller than for lateral ilium area, and not large enough for

452 comparative regressions. Allosaurus, Yangchuanosaurus, several tyrannosaurids, and

453 Ceratosaurus have sufficiently well-preserved tails. Allen et al. (2009) and Persons and Currie

454 (2011a) found that a good osteological predictor of CFL cross-sectional area is vertical distance

455 from the distal tip of the caudal ribs to the ventral tip of the haemal spines. The CFL is never

456 constrained in width to the lateral extent of the caudal ribs, as often previously reconstructed

457 (Persons and Currie 2011a). As a baseline estimate (see Discussion for caveats), we assumed the

458 maximum cross-section to be that at the deepest haemal spine, and that the cross-sections were

459 semi-circular (as ES personally observed in dissections by Persons and Currie 2011a) minus

460 cross-sections of the centra. This method unrealistically simplifies the attachments, ignoring that

461 the lateral and vertical limits of CFL origin are set by the intermucular septum on the caudal ribs

462 between CFL and m. ilioichiocaudalis (Persons and Currie 2011b). Also, simply estimating

463 cross-sections as a proxy for force overlooks functionally and ontogenetically important aspects 
464 of intramuscular anatomy, such positive allometry of fascicle length evident in the CFL of

465 Alligator mississippiensis (Allen et al. 2010). However, as with using the area of the ilium as a

466 proxy for muscle cross-section and force, using tail depth ventral to the caudal ribs is based

467 directly on fossil data. Because the articulations between the haemal arch and caudal centra

468 may not be accurate in skeletal mounts, we varied depths by $+/-10 \%$ to assess their effects on

469 CFL cross section, and on indices of turning performance. As for our tail cross-section and mass

470 estimates, we also applied the same correction factor of 1.4, that Mallison et al. (2015)

471 determined for adult Alligator, to our estimates of m. caudofemoralis cross-sections, to set an

472 upper bound for cross-section and force.

473 Estimates and comparisons of relative agility

474 We developed two indices of relative agility for theropods: Agilityforce based on

475 agility/force correlations in humans (Peterson et al. 2006, Thomas et al. 2009, Weiss et al. 2010),

476 and Agility $_{\text {moment }}$ which incorporates moments or torques. In human studies, maximal muscle

477 force relative to body mass correlates inversely with the time athletes take to complete an

478 obstacle course, which involves rapid changes of direction. Because force is a close direct

479 correlate of agility in humans, independent of torque or power, we were confident in applying

480 force to theropod agility. For Agilityforce (equation 13), we divided proxies for overall muscle

481 force (area of muscle origin on the ilium, and cross-section estimates for the $\mathrm{m}$. caudofemoralis

482 longus) by $I_{y}$, mass moment of inertia about the y axis through the axial body's center of mass

483 and a measure of the difficulty of turning the body. This is a comparative index of turning ability,

484 rather than a specific biomechanical quantity.

485 13) $\quad$ Agility $_{\text {force }}=A_{\text {ilium }} / I_{y}$ 
486 Here $A_{\text {ilium }}$ is the area $\left(\mathrm{cm}^{2}\right)$ of the ilium in lateral view. To compare this index of turning ability

487 across theropods, we plotted the results for Agilityforce against $\log 10$ of body mass for

488 tyrannosaurs and non-tyrannosaurs.

To obtain Agility moment $_{\text {, we first assumed that moment arms scale as mass }}^{1 / 3}$ (an inverse operation of Erickson and Tumanova's [2000] Developmental Mass Extrapolation). Mass ${ }^{1 / 3}$

491 approximates isometric scaling of moment arms relative to linear size of the animals, which

492 Bates et al. (2012) found to be the likely relationship for allosauroids. Applying this relationship 493 to all of the theropods, we calculated an index of comparative moments, $\tau_{\text {relative, }}$ using equation 49414 ,

14) $\tau_{\text {relative }}=\left(m^{1 / 3 / 100) \times}\right.$ Area ${ }_{\text {ilium }} \times 20 \mathrm{~N} / \mathrm{cm}^{2}$,

496 where $m$ is body mass in $\mathrm{kg}$, Area $a_{\text {ilium }}$ is ilium area in $\mathrm{cm}^{2}$, and $20 \mathrm{~N} / \mathrm{cm}^{2}$ is a sub-maximal

497 concentric specific tension (Snively and Russell 2007b). In SI units, $\mathrm{m}^{1 / 3}$ gives unrealistic

498 moment arms on the order of many meters for larger taxa. Dividing by 100 brings relative

499 moment arms into the more intuitive range of fractions of a meter. This is an arbitrary linear

500 adjustment that (1) does not imply that we have arrived at actual moment arms or torques during

501 life, and yet (2) maintains proportions of $\tau_{\text {relative }}$ among the taxa. Agility moment $\tau_{\text {relative }}$

502 divided by Iy (equation 15), which gives an index of angular acceleration.

503 15) Agility moment $_{\text {relative }} / I_{y}$

504 The quantity $\tau_{\text {relative }}$ does not use actual moment arms, and is not intended for finding 
505 angular accelerations. However, our index of relative moment arm lengths is anchored in the

506 isometric scaling of moment arms that Bates et al. (2012) found for allosauroids, and will be

507 testable with more exact estimates from modeling studies. A rich literature directly assesses

508 moment arm lengths in dinosaurs and other archosaurs (e.g. Hutchinson et al. 2005, Bates and

509 Schachner 2012, Bates et al. 2012, Maidment et al. 2013), and such methods will be ideal for

510 future studies that incorporate estimates of moment arms of individual muscles.

\section{Visualization of agility comparisons}

Although log transformation of mass is useful for statistical comparisons, plotting the raw

513 data enables intuitive visual comparisons of tyrannosaur and non-tyrannosaur agility, and

514 immediate visual identification of outliers (Packard et al. 2009). We plotted raw agility index

515 scores against $\log 10$ body mass in JMP (SAS Institute), which fitted exponential functions of

516 best fit to the data.

517 Statistical comparison of group differences: phylogenetic ANCOVA

518 Phylogenetic ANCOVA (phylANCOVA) enabled us to simultaneously test the influence

519 of phylogeny and ontogeny on agility in monophyletic tyrannosaurs versus a heterogeneous

520 group of other theropods. The phylANCOVA mathematically addresses phylogenetically distant

521 specimens or size outliers that would require separate, semi-quantitative exploration in a non-

522 phylogenetic ANCOVA.

523 Phylogenetic approach

524 All phylogenetically-inclusive analyses were conducted using the statistical program R

525 (R Core Team, 2015). For our phylogenetic framework, we used a combination of consensus

526 trees: Carrano et al. (2012) for the non-tyrannosauroid taxa (their analyses include the 
527 tyrannosauroid Proceratosaurus), and Brusatte and Carr (2016) for Tyrannosaurioidea, which

528 uses Allosaurus as an outgroup. Multiple specimens within the same species (for Tyrannosaurus

529 rex and Tarbosaurus bataar) were treated as hard polytomies (sensu Purvis and Garland, 1993;

530 Ives et al., 2007). Basic tree manipulation was performed using the \{ape\} package in $\mathrm{R}$ (version

531 3.5, Paradis et al., 2004). Branch lengths were calculated by time-calibrating the resultant tree, as

532 follows. First and last occurrences were downloaded from Fossilworks.org (see SI file for

533 Fossilworks citations). Specimens within the same species were further adjusted according to

534 their locality-specific intervals. Time calibration followed the equal-rate-sharing method of

535 Brusatte et al. (2008), which avoids zero-length branches by using a two-pass algorithm to build

536 on previously established methods (e.g. Norell, 1992; Smith, 1994; Ruta et al., 2006). This

537 arbitrarily resolved same-taxon polytomies by assigning near-zero-length branches to the base of

538 each species. The near-zero-length branches effectively maintain the hard polytomy while

539 facilitating transformations of the non-ultrametric variance-covariance matrix.

540

541

Determining strength of phylogenetic signal and appropriateness of phylogenetic regression

To determine whether phylogenetic regression was necessary when analyzing theropod

543

agility, we calculated Pagel's $\lambda$ (Pagel 1999) for each trait examined. Phylogenetic signal was

544 estimated using the R package \{phytools\} (Revell, 2012). We found that phylogenetic signal was

545 high for all traits $\left(\lambda_{\text {agility force }}=0.89 ; \lambda_{\text {agility moment }}=0.90 ; \lambda_{\text {mass }}=0.88\right)$, emphasizing the need for

546 phylogenetically-informed regression and analysis of covariance.

548 Phylogenetically informed analyses

549 A combination of phylogenetically-informed generalized least squares (PGLS) regression

550 and phylogenetic analysis of covariance (phylANCOVA) was used to test for significant 
551 deviations from allometric predictions for both agility force and agility moment (Garland et al.,

552 1993; Smaers and Rohlf, 2016). The PGLS model calculates the slope, intercept, confidence, and

553 prediction intervals following a general linear model, adjusting expected covariance according to

554 phylogenetic signal (in this case, Pagel's $\lambda$; Pagel 1999; for a recent discussion of PGLS

555 methodology, see Symonds and Blomberg 2014). PGLS regression was conducted using the R

556 package \{caper\} (Orme et al., 2013), which implements regression analysis as outlined by

557 Freckleton and colleagues (2002). We then tested for significant departures from allometry using

558 the recently-derived phylogenetic ANCOVA method of Smaers and Rohlf (2016). In standard

559 ANCOVA methodologies, comparisons are made outside of a least-squares framework (Garland

560 et al., 1993; Garland and Adolph 1994; Smaers and Rohlf, 2016). As implemented in the R

561 package \{evomap\} (Smaers, 2014), phylogenetic ANCOVA compares differences in residual

562 variance in conjunction with the phylogenetic regression parameters (Smaers and Rohlf, 2016).

563 This enables a direct least-squares test comparing the fit of multiple grades relative to a single

564 grade (Smaers and Rohlf 2016). We assigned three groups using indicator vectors:

565 Tyrannosauridae, putative juveniles within Tyrannosauridae (hereafter "juveniles"), and non-

566 tyrannosaur theropods (hereafter "other theropods"). GLS standard errors were used to directly

567 test for significant differences in intercept and slope between groups, within a generalized

568 ANCOVA framework (Smaers and Rohlf, 2016). We tested the following groupings: 1) Among

569 groups (adult Tyrannosauridae vs. juveniles vs. other theropods); 2) juveniles vs. adult

570 Tyrannosauridae; 3) Tyrannosauridae vs. other theropods. For each of these comparisons, the

571 phylANCOVA applied F-tests to partitioned group means. This analysis was performed twice:

572 once for Agility $_{\text {force }}$ and again for Agility $_{\text {moment }}$.

573 Standard for rejecting a null hypothesis of equal agilities 

standard for comparing agility results between sample groups. Reconstructing anatomy and

576 function in fossil animals has potential for many biases — including scaling errors, anatomical

577 judgment in reconstructions and digitizing, fossil incompleteness, and variation in muscle

578 anatomy. If one group appeared to have greater agility than the other, we tested the null

579 hypothesis (no difference) with conditional error probabilities $\alpha(p)$ (Berger and Sellke 1987,

580 Sellke et al. 2001), a Bayesian-derived standard appropriate for clinical trials in medicine.

581 Conditional error probabilities give the likelihood of false discoveries/false positive results

582 (Colquhoun 2014), effectively the likelihood that the null hypothesis is true, regardless of the

583 original distribution of the data. When $\mathrm{p}=0.05$ in idealized comparisons of only two groups, the

584 probability of false discoveries approaches 29\% (Colquhoun 2014). We therefore considered

585 ANCOVA group means to be definitively different if $\mathrm{p}$ was in the range of 0.001 , at which the

586 probability of a false positive is $1.84 \%$ (Colquhoun 2014). We calculated conditional error

587 probabilities $\alpha(p)$ using equation 16 (modified from Sellke et al. [2001]), which employs the

588 originally calculated $p$ value from the ANCOVA.

589

16) $\alpha(p)=\left(1+[-e p \ln (p)]^{-1}\right)^{-1}$

\section{Results}

Mass properties and comparison with other studies

592 Masses, centers of mass, and mass moments of inertia are listed in Tables 3 and 4. "Best

593 estimate" masses (Table 3) are reported for a common cross-sectional shape of terrestrial

594 vertebrates (with a superellipse exponent of 2.3). Here we report and compare individual results, 595 and compare between groups below, under the sections "Regressions of agility indices versus 
596 body mass" and "Results of phylogenetic ANCOVA". Inter-experimenter error was negligible. For

597 example, leg masses converged to within $1 \%$ when reconstructions were identically scaled, and 598 center of mass for Daspletosaurus was within $+/-0.4 \mathrm{~mm}$.

599 Volumes and masses show broad agreement between our results and those calculated in 600 other studies, such as by laser scanning of skeletal mounts (Bates et al. 2009a,b; Hutchinson et al. 601 2011) and fitting splines between octagonal hoops or more complex cross-sections. Our 602 estimates of axial body mass (not including the legs) of Acrocanthosaurus ranged from $4416 \mathrm{~kg}$ 603 (elliptical cross sections with $\mathrm{k}=2)$ to $4617 \mathrm{~kg}$ ( $\mathrm{k}=2.3$ super-ellipse exponent), compared with the $6044485 \mathrm{~kg}$ best-estimate result of Bates et al. (2009a). A slender-model body+legs mass estimate of 605 Tyrannosaurus rex specimen FMNH PR 2081 yielded 8302-8692 kg depending on superellipse 606 cross section, compared with Hartman's (2013) GDI estimate of $8400 \mathrm{~kg}$. A 13\% broader model 607 (applying the breadth of the mount's ribcage to our entire dorsal view) yielded $9131 \mathrm{~kg}$, similar 608 to Hutchinson et al.'s (2011) estimate of $9502 \mathrm{~kg}$ (their "lean" reconstruction: Hutchinson et al. 609 2011). Our largest model (Fig. 1), with an anatomically plausible $40 \%$ broader tail (Mallison eat 610 al. 2015) and 13\% broader ribcage, yielded $9713 \mathrm{~kg}$. The current study's results for the juvenile 611 Tyrannosaurus BMR 2002.4.1 vary between 575 and $654 \mathrm{~kg}$, from $-10 \%$ to $+2.3 \%$ of the $639 \mathrm{~kg}$

612 "lean model" estimate of Hutchinson et al. (2011). Volumes for Tyrannosaurus and

613 Giganotosaurus are lower than those calculated by Henderson and Snively (2003) and Therrien 614 and Henderson (2007), because leg width was narrower in the current study. However, the 615 broad-model volume estimate for the large Tyrannosaurus converges with the narrow-ribcage

616 model used in Henderson and Snively's (2003) sensitivity analysis, suggesting reasonable 617 precision given inevitable errors of reconstruction. 
619 change with the upper-bound correction factor of 1.4 times the tail cross-sectional area (Mallison

620 et al. 2015) and mass. However, absolute masses of the entire bodies increased by 5-7\% in the

621 tyrannosaurids and most allosauroids, and by 17\% in Acrocanthosaurus. With this adjustment to

622 tail cross-section, our mass estimates for the Tyrannosaurus specimens fell within the lower part

623 of the range that Hutchinson et al. (2011) calculated for the largest specimen of this taxon.

624 Centers of mass shifted posteriorly by 5-15\% (greatest for Allosaurus), placing them closer to the 625 anteroposterior location of the acetabulum. The centers of mass were anteroposteriorly

626 coincident with the acetabulum in the large-tail models of Acrocanthosaurus and Sinraptor. With

627 or without an expanded tail, the CM for Acrocanthosaurus was found to be consistent with

628 results of Bates et al. (2012), but to lie posterior to the position estimated by Henderson and 629 Snively (2003).

630 The largest specimens, Giganotosaurus carolinii and the large Tyrannosaurus rex, are

631 nearly two tonnes more massive than their nearest relatives in the sample. The adult

632 Tyrannosaurus rex specimens are more massive than Giganotosaurus carolinii, corroborating 633 predictions of Mazzetta et al. (2004) and calculations of Hartman (2013) for the specimens. The 634 axial body of the reconstructed Giganotosaurus specimen is longer, but the large legs and wide 635 axial body of the T. rex specimens contribute to a greater mass overall.

636 Changing the depth of the tails by $+/-10 \%$ changed the mass of the tails by the same 637 amount, but changed the overall body masses by no more than $3 \%$ (less in the tyrannosaurids, 638 which had more massive legs). Varying tail depth changed mass moments of inertia $I_{y}$ by less 639 than $4 \%$, too small to have an effect on trends in relative $I_{y}$ in tyrannosaurids versus non640 tyrannosaurids. 
$642 I_{y}$ of the axial bodies alone, and agilities correspondingly lower. $I_{y}$ with the swing leg increased

643 the least with Acrocanthosaurus, Giganotosaurus, large specimens of Tarbosaurus and

644 especially Tyrannosaurus, and (surprisingly) Raptorex. Gorgosaurus juveniles, with

645 proportionally long legs, showed the greatest increase in $I_{y}$ and drops in agility scores when

646 pivoting on one foot.

647 Muscle attachments and cross-sectional estimates

648 Table 3 reports ilium areas of all specimens, and Table 5 gives tail dimensions and

649 calculated cross-sectional areas for the m. caudofemoralis longus. Tyrannosaurids have 1.2-2

650 times the ilium area of other large theropods of similar mass (Table 3); these ratios increase

651 substantially when only axial body mass (total minus leg mass) is considered, because

652 tyrannosaurids have longer and more massive legs.

653 M. caudofemoralis longus cross sections vary less than ilium area between the theropods

654 (Table 5). They were slightly greater relative to body mass in most tyrannosaurids, which have

655 deeper caudal centra compared with other theropods. For example, the CFL area of the adult

656 Tyrannosaurus specimens had 1.26-1.34 times the cross-sectional areas of the Acrocanthosaurus

657 and Giganotosaurus specimens of similar respective mass. Increasing the transverse dimensions

658 of the m. caudofemoralis longus by 1.4 times, after Mallison et al. (2015), increases cross

659 sectional areas by the same factor of 1.4 because tail depth did not change. Increasing tail depth

660 by $10 \%$ predictably increased CFL area by $21 \%$, and decreasing tail depth by $10 \%$ decreased

661 CFL area by $19 \%$.

662 Regressions of agility indices versus body mass 
664 for tyrannosaurids are higher than for non-tyrannosaurids of similar body mass. Large

665 tyrannosaurids (between 2 and 10 tonnes) have at least twice the Agility force $_{\text {or }}$ Agility $_{\text {moment }}$ values 666 of the non-tyrannosaurids. For theropods in the $300-700 \mathrm{~kg}$ range, this gap increases to $2-3$ times 667 greater agility in juvenile tyrannosaurids than in allosauroid adults of similar mass. Comparing 668 specimens of different body masses, tyrannosaurids have similar agility values to those of other 669 theropods about half their size.

670 Results of phylogenetic ANCOVA

671 Across all variables, we estimated that much of theropod agility covariance structure can

672 be attributed to phylogenetic affiliation (all $\lambda>0.88$ ). The PGLS regression models indicate a 673 strong relationship between agility and mass (Figs. 4, 5), as well as low variance within agility 674 force $\left(\mathrm{R}_{\text {planted }}^{2}=0.9724 ; \mathrm{R}_{\text {pointe }}^{2}=0.9703\right)$ and agility moment $\left(\mathrm{R}_{\text {planted }}^{2}=0.9387 ; \mathrm{R}_{\text {pointe }}^{2}=\right.$ 675 0.9384). The $\lambda$-adjusted PGLS regression line under-predicts agility, fitting non-tyrannosaur 676 theropods more closely than tyrannosaurids (Figs. 4, 5), indicating that theropods as a whole are 677 more agile than predicted by phylogeny. When 95\% confidence and prediction intervals (CI and $678 \mathrm{PI}$ ) are calculated according to the phylogenetic variance structure, all tyrannosaurids at or above 679 the 95\% PI for all phylogenetic regressions (Figs. 4, 5).

680

681 Overall, phylANCOVAs for both agility force and agility moment reveal significant 682 differences among all three of our designated groups: tyrannosaurids and putative juveniles 683 versus other theropods (Tables 6 and $7 ; P_{A F \text { planted }}=0.0014 ; P_{A F \text { pointe }}=0.0013 ; P_{\text {AM planted }}=0.077$; $\left.684 P_{A M \text { pointe }}=0.0011\right)$. When the analysis was broken into specific group-wise comparisons, 685 tyrannosaurids were found to be distinctive from other theropods, whether in the context of 
686 agility force or agility moment (Tables 6 and $7 ; P_{A F \text { planted }}=0.0004 ; P_{A F \text { pointe }}=0.0005 ; P_{A M \text { planted }}$ $\left.687=0.0024 ; P_{A M \text { pointe }}=0.0004\right)$. Putative tyrannosaurid juveniles were not found to be significantly 688 different than their adult counterparts for either performance metric $\left(\right.$ Tables 6 and $7 ; P_{A F \text { planted }}=$ $\left.6890.9421 ; P_{A F \text { pointe }}=0.7044 ; P_{A M \text { planted }}=0.9301 ; P_{\text {AM pointe }}=0.6938\right)$. For this reason, juveniles are 690 not considered apart from adults and have a similar relationship between mass and agility.

691 Conditional error probabilities $\alpha(p)$ for comparisons between groups (Tables 6 and 7) range from $6920.008\left[\alpha(p)_{A F \text { planted }}\right.$ and $\left.\alpha(p)_{\text {AM pointe }}\right]$ to $0.038\left[\alpha(p)_{\text {AM planted }}\right]$, indicating low to negligible 693 probabilities of false positive results. Conditional error probability among groups was high for $694 \alpha(p)_{A M \text { planted }}$, possibly because moment arms scale with mass ${ }^{1 / 3}$ and have a great influence on the 695 outcome. However, $\alpha\left(p\right.$ are still low for both Agility $_{\text {moment }}$ comparison between all tyrannosaurids 696 vs other theropods.

697 Discussion

698 Phylogenetic ANCOVA demonstrates definitively greater agility in tyrannosaurids relative to 699 other large theropods examined.

700 Regressions of agility indices against body mass (Figs. 3-5), and especially phylogenetic 701 ANCOVA (Figs. 4, 5), corroborate the hypotheses that tyrannosaurids could maneuver more 702 quickly than allosauroids and some other theropods of the same mass.

703 To evaluate potential biologically-relevant distinctiveness between tyrannosaurids and 704 other theropods, we used a recently developed method of phylogenetic ANCOVA that enabled 705 group-wise comparisons in the context of the total-group covariance structure (Smaers and Rohlf, 706 2016). By preserving the covariance structure of the entire dataset, this method yields a more 707 appropriate hypothesis test for comparing groups of closely related species (as compared to 708 standard ANCOVA procedures which segregate portions the dataset and therefore compare 
709 fundamentally different covariance structures; Garland et al., 1993; Garland and Adolph, 1994).

710 Our phylogenetic regression analysis finds that agility and mass are strongly correlated among

711 all theropods $\left(\mathrm{R}^{2}>0.94 ; P<0.001\right)$, and exhibit a high degree of phylogenetic signal $(\lambda>0.88)$.

712 Using the phylANCOVA of Smaers and Rohlf (2016), we were able to determine that

713 tyrannosaurids exhibit significantly higher agility metrics than other theropods (Figs. 3-5; Tables

7146 and 7). Putative tyrannosaurid juveniles were not found to be significantly different from adults

715 and were on or within the $95 \%$ prediction interval, aligning these individuals closer to expected

716 phylogenetic structure of their adult counterparts (Figs. 4, 5; Tables 6 and 7). The slope of the

717 phylogenetic regression lines are greater than -1 but less than 0 , suggesting that agility decreases

718 out of proportion to mass as theropods grow, likely because $I_{y}$ is proportional to the square of

719 body length (Henderson and Snively 2003).

720 These results allow us to draw important evolutionary conclusions, highlighting the

721 possibility of locomotor niche stratification within Theropoda. The strength of phylogenetic

722 signal combined with the clear degree of separation between tyrannosaurids and non-tyrannosaur

723 theropods underscore the importance of using a phylogenetically-informed ANCOVA to

724 understand between- and within-group agility evolution. By using a phylogenetically-informed

725 analysis, we are able to confirm significant differences in turning behavior, with tyrannosaurs

726 possessing uniquely superior agility scores. These results could indicate a functional

727 specialization for distinctive ecological niches among these groups.

728 Studies of performance evolution can be difficult because morphology does not always

729 translate into performance differences (Garland and Losos, 1994; Lauder, 1996; Lauder and

730 Reilly, 1996; Irschick and Garland, 2001; Toro et al., 2004). This study, through quantification

731 of multi-body, multifaceted performance metrics, finds strong relationships between morphology, 
732 agility, and a distinctive performance capacity by tyrannosaurids. With respect to other theropods,

733 tyrannosaurids are increasingly agile without compromising their large body mass, such that in a

734 pairwise comparison, tyrannosaurids are achieving the same agility performance of much smaller

735 theropods (Figs. 3-5). For example, a $500 \mathrm{~kg}$ Gorgosaurus has slightly greater agility scores than

736 the $200 \mathrm{~kg}$ Eustreptospondylus, and an adult Tarbosaurus nearly twice the agility scores of the

737 lighter Sinraptor This agility performance stratification suggests that these two groups may have

738 had different ecologies, inclusive of both feeding and locomotory strategies. Further, by

739 including juveniles in our analysis through the use of independent inclusion vectors, we were

740 further able to estimate performance capacity in younger life history stages. This revealed that

741 agility performance is established relatively early in life and carries through to large adult body

742 masses.

743 This quantitative evidence of greater agility in tyrannosaurids is robust, but requires the

744 consideration of several caveats. Agility scores rest on the relationships between agility and

745 muscle force, and muscle force and attachment area. Muscle force and agility correlate directly

746 with each other in humans (Peterson et al. 2006, Thomas et al. 2009, Weiss et al. 2010), and at a

747 gross level muscle cross-sectional area and force scale with the size of muscle attachments

748 (Snively and Russell 2007a). However, these correlations have yet to be studied in the same

749 system, for example linking ilium area to force and agility in humans. More thorough testing of

750 the hypothesis will require detailed characterization of muscle sizes, forces and moments in

751 theropods (Hutchinson et al. 2007, 2011). However, based on dramatic and statistically robust

752 differences between tyrannosaurids and other theropods (Figs. 3-6), we predict that refined

753 studies will corroborate discrepancies in relative agility. Furthermore, we predict that with the

754 same methods, the short-skulled, deep-tailed abelisaurids will have agility indices closer to those 
755 of tyrannosaurids than to the representatives of the predominantly allosauroid sample we 756 examined.

757 Theropod mass property estimates are consistent between diverse methods, suggesting reliable 758 inferences about relative agility.

759 Theropod mass and $I_{y}$ estimates in this study converge with those of other workers, 760 despite differing reconstructions and methods. Our mass estimates for one large Tyrannosaurus 761 rex (FMNH PR 2081) are within + or - 6\% of the "lean" estimate of Hutchinson et al. (2011),

762 who laser scanned the mounted skeleton with millimeter-scale accuracy. Hutchinson et al.'s

763 (2011) models of this specimen probably have more accurate dorsoventral tail dimensions than

764 ours, with a relatively greater depth corresponding to that of extant sauroposids (Allen et al.

765 2009), whereas our models have broader tails. Our mass estimate for the "Jane" specimen (BMR

766 2002.4.1) was similarly close. These convergences are remarkable, considering that we

767 conducted our estimates long before we were aware of this parallel research, and using a

768 different method. Depending on assumed cross-sections, our axial body estimates for

769 Acrocanthosaurus ranged from $-1.6 \%$ to $+2.9 \%$ of those of Bates et al. (2009b), which were

770 obtained from laser scanning for linear dimensions, and lofted computer models for volume. As

771 for our estimates of Tyrannosaurus mass properties, the Acrocanthosaurus calculations were

772 "blind" to Bates et al.'s (2009a) results for this specimen. For all of the examined taxa, volumes

773 of the neck and width of the base of the tail are likely greater in our study than in others, even

774 with robust models in their sensitivity analyses (Hutchinson et al. 2007; Bates et al. 2009a,b),

775 because our models incorporate new anatomical data on soft tissues (Snively and Russell 2007b,

776 Allen et al. 2009, Persons and Currie 2010, Mallison et al. 2015) indicating a taller, broader neck

777 and broader tail cross-sections. Despite these discrepancies in soft tissue reconstruction, high 
778 consistency with methods based on scanning full-sized specimens engenders optimism about the

779 validity of frustum-method estimates (Henderson 1999), despite their dependence on 2D images,

780 restoration accuracy, and researcher judgments about amounts of soft tissue.

781 Frustum and graphical double integration (GDI) methods also yielded similar results

782 (Appendix 1). When superellipse correction factors were applied to the $9.2 \mathrm{~m}^{3}$ GDI volume

783 Hartman (2013) obtained for the Tyrannosaurus rex (PR 2081), results closer to our broad-

784 bodied volume estimate for the specimen were generated. Assuming a super-ellipse exponent of

785 2.3, scaling Hartman's (2013) estimate by the correction factor of 1.047 gives an estimate of

$7869.632 \mathrm{~m}^{3}$, less than $2 \%$ greater than our estimate. Furthermore, applying super-ellipsoid cross

787 sections may reconcile careful GDI estimates, such as Taylor's (2009) for the sauropods

788 Brachiosaurus and Giraffatitan, with volumes evident from laser scans and photogrammetry of

789 fossil mounts (Gunga et al. 2008, Bates et al. 2016).

790 In addition to convergence of mass and volume estimates, different algorithms for center

791 of mass give nearly identical COM estimates for Giganotosaurus, the longest theropod in the

792 sample (see Appendix 1). The discrepancy of only $0.2 \mathrm{~mm}$ is negligible for a $13 \mathrm{~m}$-long animal.

793 Although we recommend finding the anteroposterior COM of each frustum using our equation 4

794 (especially for rotational inertia calculations), the simpler approximation method is adequate.

795 Calculation methods probably have a smaller effect on center of mass estimates than

796 anatomical assumptions concerning restoration, and variations in the animal's postures in real

797 time. Such postural changes would include turning or retracting the head, and movements of the

798 tail (Carrier et al. 2001) using axial (Persons and Currie 2011a, b; Persons and Currie 2013) and

799 caudofemoral muscles (Bates et al. 2009; Allen et al. 2010; Persons and Currie 2011a, b;

800 Hutchinson et al. 2011; Persons and Currie 2013). The congruence of results from different 
801 methods is encouraging, because biological factors govern the outcome more than the choice of

802 reconstruction method.

803 Relative agilities are insensitive to modeling bias.

804 Reconstruction differences between this and other studies are unlikely to bias the overall 805 comparative results so long as anatomical judgments and methods are consistently applied to all

806 taxa. For example, although tail width is reconstructed similarly in this study and the dissection-

807 based studies of Allen et al. (2010) and Persons and Currie (2011), the tail depths of our models

808 may be too shallow (Allen et al. 2010). Consistently deeper tails, better matching reconstructions

809 of Allen et al. (2010), Bates et al. (2009a, b) and Hutchinson et al. (2011), would, however, not

810 alter our overall comparative results.

811 Considering $I_{y}$ and mass from independent studies is instructive in relation to potential

812 modeling bias and error. Bates et al. (2009b) calculated notably high mass and $I_{y}$ (Hutchinson et

813 al. 2011) for a Tyrannosaurus rex specimen (MOR 555) not included in our study, yet with its

814 enormous ilium its agility indices would be higher than those of a non-tyrannosaurid

815 Acrocanthosaurus of equivalent mass (Bates et al. 2009b). $I_{y}$ and agility for the Allosaurus

816 examined by Bates et al. (2009a) are similar to those for other Allosaurus specimens. Consistent

817 modeling bias for all theropods (making them all thinner or more robust) would have no effect

818 on relative agility assessments. Overlap of agility would require inconsistent bias in this study

819 and those of other workers, with more robust tyrannosaurid reconstructions and slender non-

820 tyrannosaurids. This bias is unlikely, because reconstructions were checked against skeletal

821 measurements and modified when necessary, and most reconstructions were drawn from one

822 source (Paul 2010). 
824 methods based on limb circumference-to-mass scaling in bipeds. Our lower mass estimate (6976

$825 \mathrm{~kg}$ ) for one adult Tyrannosaurus rex specimen (AMNH 5027) coincides remarkably with their

826 results $(6688 \mathrm{~kg})$, considering the large tail width of our reconstruction. These close

827 correspondences of inertial properties between different studies gives confidence for biological

828 interpretation.

829 Behavioral and ecological implications of agility in large theropods

830 This discrepancy in agility between tyrannosaurids and other large theropods raises

831 specific implications for prey preference, hunting style, and ecology. By being able to maneuver

832 faster, tyrannosaurids were presumably more adept than earlier large theropods in hunting

833 relatively smaller (Hone and Rauhut 2009), more agile prey, and/or prey more capable of active

834 defense. This capability in tyrannosaurids is consistent with coprolite evidence that indicates

835 tyrannosaurids fed upon juvenile ornithischians (Chin et al. 1998, Varricchio 2001), and with

836 healed tyrannosaurid bite marks on adult ceratopsians and hadrosaurs (Carpenter 2000,

837 Wegweiser et al. 2004, Happ 2008). Tyrannosaurids co-existed with herbivorous dinosaurs that

838 were predominately equal to or smaller than them in adult body mass. The largest non-

839 tyrannosaurids, including Giganotosaurus, often lived in habitats alongside long-necked

840 sauropod dinosaurs, the largest land animals ever. These associations suggest that allosauroids

841 may have preferred less agile prey than did tyrannosaurids. It is also possible that stability

842 conferred by high rotational inertia, as when holding onto giant prey, was more important for

843 allosauroids than turning quickly.

844 These faunal correspondences between predator agility and adult prey size are not

845 absolute, however. Tyrannosaurids sometimes shared habitats with large sauropods (Nemegt, 
846 Ojo Alamo, and Javalina Formations: Borsuk-Białynicka 1977, Lehman and Coulson 2002,

847 Sullivan and Lucas 2006, Fowler and Sullivan 2011), and even with exceptionally large

848 hadrosaurids (Hone et al. 2014). Relative agility of herbivorous dinosaurs must be tested

849 biomechanically to assess the possible advantages of agility in tyrannosaurids. Snively et al.

850 (2015) calculated that ceratopsians had lower $I_{y}$, and hadrosaurs and sauropods greater $I_{y}$, than

851 contemporaneous theropods, but musculoskeletal turning ability has yet to be assessed in detail 852 for dinosaurian herbivores.

853 Tyrannosaurids were unusual in being the only toothed theropods (thus excluding large-

854 to-giant oviraptorosaurs and ornithomimosaurs) larger than extant wolves in most of their

855 habitats (Farlow and Holtz 2002, Farlow and Pianka 2002, Holtz 2004). Among toothed

856 theropods, adult tyrannosaurids of the Dinosaur Park Formation were 50-130 times more

857 massive than the next largest taxa (troodontids and dromaeosaurids: Farlow and Pianka 2002).

858 Comparing the dromaeosaur Dakotaraptor steini (DePalma et al. 2015) and Tyrannosaurus rex

859 in the Hell Creek formation reveals an instructive minimum discrepancy. We estimate the mass

860 of Dakotaraptor to be $374 \mathrm{~kg}$, using the femoral dimensions provided by DePalma et al. (2015:

861 Fig. 9) and the equations of Campione et al. (2014). Adult Tyrannosaurus attained 17-24 times

862 this mass (our estimates), approximately the difference between a large male lion and an adult

863 black backed jackal. By our estimates, the juvenile Tyrannosaurus in our sample was nearly

864 twice as massive as an adult Dakotarapor.

865 These size differences between adult tyrannosaurids and non-tyrannosaurid predators

866 suggest that subadult tyrannosaurids were able to capably hunt midsized prey, in ecological roles

867 vacated by less-agile, earlier adult theropods of similar body mass. In contrast, many earlier

868 faunas (Foster et al. 2001, Farlow and Holtz 2002, Farlow and Pianka 2002, Russell and Paesler 
869 2003, Holtz 2004, Foster 2007, Läng et al. 2013; although see McGowen and Dyke 2009) had a

870 continuum of body masses between the largest and smallest adult theropods, and perhaps greater

871 subdivision of niches between adults (Läng et al. 2013). A companion paper (Surring et al., in

872 revision) explores alternative evolutionary scenarios, and presents soft-tissue evidence in a

873 further exploration of tyrannosaurid agility.

874 Appendix

875 How precise are different methods of mass property estimation?

876 In addition to our mathematical slicing procedures (Henderson 1999), methods for

877 calculating mass properties include use of simplified B-splines or convex hulls to represent body

878 regions (Hutchinson et al. 2007, Sellers et al. 2012, Brassey and Sellars 2014, Brassey et al.

879 2016), or more complex NURBS (non-uniform rational B-spline) reconstruction modified to fit

880 the contours of mounted skeletons and inferred soft tissues (Bates et al. 2009a, b; Mallison 2007,

881 2010, 2014; Stoinsky et al. 2011). Brassey (2017) reviews and compares these methods in detail.

882 Both spline-based and mathematical slicing methods have been validated for living terrestrial

883 vertebrates (Henderson 1999, 2004, 2006; Henderson and Snively 2003, Hutchinson et al. 2007,

884 Bates et al. 2009a). However, spline-based methods [as in Mallison's (2007, 2010, 2014) and

885 similar procedures] are conceivably more accurate than slicing methods, which are based on a

886 few extreme coordinates of the body, and estimate intermediate contours as ellipses or non-

887 ellipsoid superellipses (Henderson 1999, Motani 2001, Henderson and Snively 2003, Arbour

888 2009, Snively et al. 2013). We compared results of mathematical slicing and spline methods by

889 obtaining inertial properties from both slicing abstractions and spline models of several

890 theropods, based on the dimensions used in the slicing calculations. 
892 cylinders instead of frusta to estimate volumes. For reptiles with cylindrical bodies, GDI

893 approximates mass better than regressions based on body length or bone dimensions (Hurlburt 894 1999). Masses and Iy were calculated by GDI for all specimens, and compared to results from 895 the frustum method.

896 Methods for testing precision of mass property results from different approaches

897 To compare slicing and spline-based inertial property results of full axial bodies of

898 theropods, we constructed spline models of Yangchuanosaurus shangyouensis, Sinraptor 899 hepingensis, and Tarbosaurus bataar (Fig. 6), after Snively et al. (2013) and Snively et al. 900 (2015). We used FreeCAD (freecadweb.org) to construct the bodies from lofted ellipses, and 901 MeshLab (meshlab.sourceforge.net) to obtain volume, centers of mass, and the inertia tensor, 902 assuming uniform densities.

903 We further estimated volumes of Eustreptospondylus oxoniensis and Yanchuanosaurus

904 shangyouensis using the graphical double integration methods of Jerison (1973), Hurlburt (1999),

905 Murray and Vickers Rich (2004), and Taylor (2009), using equation 12.

906 12) $\quad V_{\text {body }}=\sum_{n=1}^{i} V_{i}=\pi\left(r_{i 1}\right)\left(r_{i 2}\right) L_{i}$

907 The body is divided into segments from 1 to $\mathrm{i}$. Each body segment is treated as an elliptical 908 cylinder with the cross sectional area of its anterior ellipse, with major and minor radii of $r_{1}$ and $909 r_{2}$. This area is multiplied by L, the segment's length as the distance to the subsequent ellipse.

910 We also tested convergence of body COM approximations using COM of each frustum

911 (equation 4), versus simply assuming that each frustum's anterioposterior COM was very close

912 to its larger-diameter face. The longest specimen, Giganotosaurus carolinii, was the best

913 candidate for this test because $I_{y}$ is sensitive to the square of the distance $r$ (equation 8 ) of a 
914 segment's COM from the body total COM. The distance of the large-diameter face from the

915 animal's rostrum was used as the value for $C O M_{\text {frustum }}$ in equation 7.

916 Results of methods comparison

917 Values of mass and mass moment of inertia varied little between methods using frusta

918 (truncated cones), extruded ellipses (GDI), and spline (3D lofting) methods. Volumes, COM, and

$919 I_{y}$ (assuming uniform density) were within $0.5 \%$ of each other for frustum and spline models of

920 Sinraptor hepingensis, Yangchuanosaurus shangyouensis, and Tarbosaurus bataar (Fig. 6). The

921 GDI mass and $I_{y}$ for Eustreptospondylus oxoniensis were only $0.1 \%$ higher than calculated by the

922 frustum method, and that for Yanchuanosaurus shangyouensis only $0.5 \%$ higher. However,

923 differences increase substantially for estimates of hind limb mass. GDI-calculated mass for the

924 hind leg of Eustreptospondylus is over $11 \%$ greater than that from the frustum method.

925 GDI and frustum estimates are closest for axial bodies of the theropods, but diverged for

926 the hind legs. This suggests high accuracy of the method for relatively tubular objects, such as

927 the bodies of some sprawling tetrapods (Hurlburt 1999), and the necks, tails, and legs of giant

928 long-necked sauropod dinosaurs (Taylor 2009). GDI with extruded ellipses is less accurate for

929 highly tapered objects, such as the hind legs of theropods, the trunks of some large theropods and

930 sauropods, and other animals with ribcages that flare laterally in coronal section. However, the

931 high frequency of body cross sections (Motani 2001), as in our axial body models, ameliorates

932 the potential error of GDI for tapered objects.

933 For the Giganotosaurus model, the position of $C O M_{b o d y}$ from the tip of the rostrum was

934 identical to three significant figures, whether using equation 4 or assuming that each frustum's

935 COM was very close to its larger face $\left(4.65665 \mathrm{~m}\right.$ versus $4.65685 \mathrm{~m}$, a difference of $\left.2 \times 10^{-4} \mathrm{~m}\right)$. 936 


\section{References}

941

942

943

944

945

946

947

948

949

950

951

952

953

954

955

956

957

958

Allen V., Paxton H., and Hutchinson J.R. 2009. Variation in center of mass estimates for extant sauropsids and its importance for reconstructing inertial properties of extinct archosaurs.

Anatomical Record 292: 1442-1461.

Anderson M.A., Gieck J.H., Perrin D., Weltman A., Rutt R., and Denegar C. 1991. The

relationships among isometric, isotonic, and isokinetic concentric and eccentric quadriceps and hamstring force and three components of athletic performance. Journal of Orthopaedic \& Sports Physical Therapy 14: 114-120.

Arbour V.M. 2009. Estimating impact forces of tail club strikes by ankylosaurid dinosaurs. PLoS ONE 4(8): e6738. doi:10.1371/journal.pone.0006738

Bakker R.T. and Bir G. 2004. Dinosaur crime scene investigations: theropod behavior at Como Bluff, Wyoming, and the evolution of birdness. In: Currie P.J., Koppelhus E.B., Shugar M.A., and Wright J.L. (eds). Feathered dragons: Studies on the Transition from Dinosaurs to Birds. pp. 301-342. Indiana University Press, Bloomington.

959

960

Bates K.T., Falkingham P.L., Breithaupt B.H., Hodgetts D., Sellers W.I., and Manning P.L.

961

962

963

964 2009a. How big was 'Big Al'? Quantifying the effect of soft tissue and osteological unknowns on mass predictions for Allosaurus (Dinosauria:Theropoda). Palaeontologia Electronica Vol. 12, Issue 3; 14A: 33p; http://palaeo-electronica.org/2009_3/186/index.html

965

966

967

Bates K.T., Manning P.L., Hodgetts D., and Sellers W.I. 2009b. Estimating mass properties of dinosaurs using laser imaging and 3D computer modelling. PLoS ONE 4(2): e4532.

968 doi:10.1371/journal.pone.0004532

969

970 Bates K.T., Mannion P.D., Falkingham P.L., Brusatte S.L., Hutchinson J.R., Otero A., Sellers W.I., Sullivan C., Stevens K.A., and Allen V. 2016. Temporal and phylogenetic evolution of the

972

973 sauropod dinosaur body plan. Royal Society Open Science 3: 150636.

974

975

Bates K.T. and Schachner E.R. (2012). Disparity and convergence in bipedal archosaur

976 locomotion. Journal of the Royal Society Interface 9: 1339-1353.

977

978

Bates K.T., Benson R.B.J., and Falkingham P.L. 2012. A computational analysis of locomotor

979 anatomy and body mass evolution in Allosauroidea (Dinosauria: Theropoda). Paleobiology 38: 
980

981

982

983

984

985

986

987

988

989

990

991

992

993

994

995

996

997

998

999

1000

1001

1002

1003

1004

1005

1006

1007

1008

1009

1010

1011

1012

1013

1014

1015

1016

1017

\section{6-507.}

Berger J.O. and Sellke T. 1987 Testing a point null hypothesis: the irreconcilability of p-values and evidence. Journal of the American Statistical Association 82: 112-122.

Borsuk-Białynicka M.M. 1977. A new camarasaurid sauropod Opisthocoelicaudia skarzynskii gen. n., sp. n. from the Upper Cretaceous of Mongolia. Acta Palaeontologia Polonica 37:5-64

Brassey C.A. 2017. Body-mass estimation in paleontology: a review of volumetric techniques. The Paleontological Society Special Papers 22:133-156.

Brassey C.A. and Sellers W.I. 2014. Scaling of convex hull volume to body mass in modern primates, non-primate mammals and birds. PLoS ONE 9(3): e91691.

Brassey C.A., O’Mahoney T.G., Kitchener A.C., Manning P.L., and Sellers W.I. 2016. Convexhull mass estimates of the dodo (Raphus cucullatus): application of a CT-based mass estimation technique. PeerJ 4: e1432

Brochu C.A. 2002. Osteology of Tyrannosaurus rex: insights from a nearly complete skeleton and high-resolution computed tomographic analysis of the skull. Society of Vertebrate Paleontology Memoir 7. Journal of Vertebrate Paleontology 22 (Supplement to 4): 1-138.

Brusatte S.L. and Carr T.D. 2016. The phylogeny and evolutionary history of tyrannosauroid dinosaurs. Scientific Reports 6: 20252.

Brusatte S.L., Norell M.A., Carr T.D., Erickson G.M., Hutchinson J.R., Balanoff A.M., Bever G.S., Choiniere J.N., Makovicky P.J., and Xu X. 2010. Tyrannosaur paleobiology: new research on ancient exemplar organisms. Science.329: 1481-1485.

Calvo, J.O. and Coria, R.A. 1998. New specimen of Giganotosaurus carolinii (Coria and Salgado, 1995), supports it as the largest theropod ever found. In: Pérez-Moreno B.P., Holtz T.R.,Jr., Sanz J.L. and Moratalla J.J. (eds.). Aspects of Theropod Palaeobiology, Gaia Special Volume. pp. 117-122.

Campione N.E., Evans D.C., Brown C.M., and Carrano M.T. 2014. Body mass estimation in non-avian bipeds using a theoretical conversion to quadruped stylopodial proportions. Methods in Ecology and Evolution 5: 913-923.

Carrano M.T. and Hutchinson J.R. 2002. Pelvic and hindlimb musculature of Tyrannosaurus rex (Dinosauria: Theropoda). Journal of Morphology 253: 207-228.

Carrano M.T., Benson R.B.J., and Sampson S.D. 2012. The phylogeny of Tetanurae (Dinosauria: Theropoda). Journal of Systematic Palaeontology 10: 211-300. 
1018 Carrier D.R., Walter R.M., and Lee D.V. 2001. Influence of rotational inertia on turning

1019 performance of theropod dinosaurs: clues from humans with increased rotational inertia. Journal

1020 of Experimental Biology 204: 3917-3926.

1021

1022

1023

Chin K., Tokaryk T.T., Erickson G.M., and Calk L.C. 1998 A king-sized theropod coprolite.

1024

1025

1026

1027

1028

1029 Nature 393: 680-682.

Colquhoun D. 2014 An investigation of the false discovery rate and the misinterpretation of $p$ values. Royal Society Open Science 1: 140216.

Coria R.A. and Currie P.J. 2003. The braincase of Giganotosaurus carolinii (Dinosauria:

Theropoda) from the Upper Cretaceous of Argentina. Journal of Vertebrate Paleontology 22:

1030

1031

1032 802-811.

1033

DePalma R.A., Burnham D.A., Martin L.D., Larson P.L., and Bakker R.T. 2015. The first giant raptor (Theropoda: Dromaeosauridae) from the Hell Creek Formation. Paleontological

1034

Contributions 14: 1-16.

1035

1036

1037

Dong Z., Zhou S., and Zhang Y. 1983. Dinosaurs from the Jurassic of Sichuan. Palaeontologica Sinica New Series C 162: 1-136.

1038

1039

Durkin J. 2003. Development of a geometric modeling approach for human body segment

1040

1041

1042

1043

1044

1045

1046

1047 inertial parameters. Doctoral Thesis. McMaster University.

Erickson G.M. and Tumanova T.A. 2000. Growth curve of Psittacosaurus mongoliensis Osborn (Ceratopsia: Psittacosauridae) inferred from long bone histology. Zoological Journal of the Linnean Society 130: 551-566.

Farlow J.O. and Holtz T.R. Jr. 2002. The fossil record of predation in dinosaurs. In: Kowalewski, M. and P.H. Kelley (eds.). The Fossil Record of Predation. pp. 251-266 (The Paleontological

1048 Society Papers 8, 2002).

1049

1050

1051

Farlow J.O. and Pianka E.R. 2002. Body size overlap, habitat partitioning and living space requirements of terrestrial vertebrate predators: implications for the paleoecology of large

1052

1053

1054

1055

1056

1057

1058

1059 theropod dinosaurs. Historical Biology 16: 21-40.

Foster J. 2007. Jurassic West. Indiana University Press, Bloomington.

Foster J.R. and Chure D.J. 2006. Hindlimb geometry in the Late Jurassic theropod dinosaur Allosaurus, with comments on its abundance and distribution. In: Foster J.R. and Lucas S.G. (eds.), Paleontology and Geology of the Upper Jurassic Morrison Formation. New Mexico Museum of Natural History and Science Bulletin 36: pp. 119-122.

1060

Foster J.R., Holtz T.R. Jr., and Chure D.J. 2001. Contrasting patterns of diversity and community 1062 structure in the theropod faunas of the Late Jurassic and Late Cretaceous of Western North 1063 America. Journal of Vertebrate Paleontology 21(Supplement to 3): 51A. 
1064

1065

1066

1067

1068

1069

1070

1071

1072

1073

1074

1075

1076

1077

1078

1079

1080

1081

1082

1083

1084

1085

1086

1087

1088

1089

1090

1091

1092

1093

1094

1095

1096

1097

1098

1099

1100

1101

1102

1103

1104

1105

1106

1107

1108

1109
Fowler D.W. and Sullivan R.M. 2011. The first giant titanosaurian sauropod from the Upper Cretaceous of North America. Acta Palaeontologica Polonica 56: 685-690.

Freckleton R.P. 2002. On the misuse of residuals in ecology: Regression of residuals vs. multiple regression. Journal of Animal Ecology 71: 542-545.

Gao Y. 1992. Yanchuanosaurus hepingensis-a new species of carnosaur from Zigong, China. Vertebrata Palasiatica 32: 313-324.

Garland T. and Adolph S.C. 1994. Why not to do 2-species comparative studies-limitations on inferring adaptation. Physiologial Zoology 67: 797-828.

Garland T. Jr. and Losos J.B. 1994. Ecological morphology of locomotor performance in squamate reptiles. In: Wainwright P.C. and Reilly S. (eds.), Ecological Morphology: Integrative Organismal Biology. University of Chicago Press Chicago. pp. 240-302

Garland T., Dickerman A.W., Janis C.M., and Jones J.A. 1993. Phylogenetic analysis of covariance by computer simulation. Systematic Biology 42: 265-292.

Gilmore, C. W. 1920. Osteology of the carnivorous Dinosauria in the United States National Museum, with special reference to the genera Antrodemus (Allosaurus) and Ceratosaurus. Bulletin of the United States National Museum 110: 1-154.

Gunga H.-C., Suthau T., Bellmann A., Stoinski S., Friedrich A., Kirsch K., and Hellwich O., 2008. A new body mass estimation of Brachiosaurus brancai Janensch, 1914 mounted and exhibited at the Museum of Natural History (Berlin, Germany). Fossil Record 11: 33-38.

Harris J.D. 1998. A reanalysis of Acrocanthosaurus atokensis, its phylogenetic status, and paleobiogrographic implications, based on a new specimen from Texas. New Mexico Museum of Natural History and Science Bulletin 13: pp. 1-75.

Hartman S. 2011. Skeletal Drawing. http://skeletaldrawing.com. Accessed August 19, 2011.

Henderson D.M. 1999. Estimating the masses and centers of mass of extinct animals by 3-D mathematical slicing. Paleobiology 25: 88-106.

Henderson D.M. and Snively E. 2003. Tyrannosaurus en pointe: Allometry minimized rotational inertia of large carnivorous dinosaurs. Proceedings of the Royal Society B, Biology Letters 271: S57-S60.

Holtz T. R., Jr. 2004. Taxonomic diversity, morphological disparity, and guild structure in theropod carnivore communities: implications for paleoecology and life history strategies in tyrant dinosaurs. Journal of Vertebrate Paleontology 24(supplement to 3), 72A-72A. 
1110 Holtz T.R. Jr. 2002. Theropod predation: evidence and ecomorphology. In Predator-Prey

1111 Interactions in the Fossil Record (eds Kelly, P.H., Koweleski, M., and Hansen T.A) Topics in

1112 Geobiology 17. 325-340 (Kluwer/Plenum, 2002).

1113

1114 Holtz T.R., Jr. 1995. The arctometatarsalian pes, an unusual structure of the metatarsus of

1115 Cretaceous Theropoda (Dinosauria: Saurischia). J. Vertebr. Paleontol. 14: 480-519.

1116

1117 Hone D.W.E., Wang K., Sullivan C., Zhao X., Chen S., Li D., Ji S., Ji Q., and Xu X. 2011. A

1118 new, large tyrannosaurine theropod from the Upper Cretaceous of China. Cretaceous Research

1119 32: 495-503.

1120

1121

1122

1123

1124

1125

1126

1127

1128

1129

Hone D.W.E., Sullivan C, Zhao Q., Wang K., and Xu X. Body size distribution in a death assemblage of a colossal hadrosaurid from the Upper Cretaceous of Zhucheng, Shandong Province, China. In: Eberth D.A. and Evans D.C. (eds.). Hadrosaurs. Indiana University Press, Bloomington. pp. 524-531.

1130

Hurlburt G. 1999. Comparison of body mass estimation techniques, using recent reptiles and the pelycosaur Edaphosaurus boanerges. Journal of Vertebrate Paleontology 19: 338-350.

Hurum J.H. and Sabath K. 2003. Giant theropod dinosaurs from Asia and North America: skulls

1131 of Tarbosaurus bataar and Tyrannosaurus rex compared. Acta Palaeontologia Polonica 48:161190.

1132

1133

Hutchinson J.R., Anderson F.C., Blemker, S.S., and Delp S.L. 2005 Analysis of hind limb

1134 muscle moment arms in Tyrannosaurus rex using a three-dimensional musculoskeletal computer

1135 model: implications for stance, gait and speed. Paleobiology 31: 676 - 701

1136

1137

Hutchinson J.R., Bates K.T., Molnar J., Allen V., and Makovicky P.J. 2011. A computational

1138

1139 analysis of limb and body dimensions in Tyrannosaurus rex with implications for locomotion, ontogeny, and growth. PLoS One 6(10): e26037. doi:10.1371/journal.pone.0026037.

1140

1141

Hutchinson J.R., Ng-Thow-Hing V., and Anderson F.C. 2007. A 3D interactive method for

1142

1143

1144

1145

1146

1147

1148

1149

1150

1151

1152

1153

1154 estimating body segmental parameters in animals: Application to the turning and running performance of Tyrannosaurus rex. Journal of Theoretical Biology 246: 660-680.

Huwaldt J.A. 2010. Plot Digitizer. http://plotdigitizer.sourceforge.net. Accessed August 19, 2011.

Irschick D.J. and Garland Jr, T. 2001. Integrating function and ecology in studies of adaptation: investigations of locomotor capacity as a model system. Annual Review of Ecology and Systematics. pp.367-396.

Jerison H.J. 1973. Evolution of the brain and intelligence. Academic Press. New York.

Läng E., Boudad L., Maio L. Samankassou E., Tabouelle J., Tong H., and Cavin L. 2013. Unbalanced food web in a Late Cretaceous dinosaur assemblage. Palaeogeography,

1155 Palaeoclimatology, Palaeoecology 381-382: 26-32. 
1156

1157

1158

1159

1160

1161

1162

1163

1164

1165

1166

1167

1168

1169

1170

1171

1172

1173

1174

1175

1176

1177

1178

1179

1180

1181

1182

1183

1184

1185

1186

1187

1188

1189

1190

1191

1192

1193

1194

1195

1196

1197

1198

1199

1200

1201

Lauder, G. V. 1996. The argument from design. In: Rose M.R. and Lauder G.V. (eds.), Adaptation. Academic Press. San Diego. pp. 187-220.

Lauder G.V. and Reilly S.M. 1996. The mechanistic basis of behavioral evolution: Comparative analysis of muscoskeletal function. In: Martins (ed.), Phylogenies and the Comparative Method in Animal Behavior. Oxford University Press. Oxford. pp. 105-137.

Lehman T.M. and Coulson A.B. 2002. A juvenile specimen of the sauropod Alamosaurus sanjuanensis from the Upper Cretaceous of Big Bend National Park, Texas. Journal of Palaeontology. 7:156-172.

Li D., Norell M.A., Gao K.-Q., Smith N.D., and Makovicky P.J. 2009. A longirostrine tyrannosauroid from the Early Cretaceous of China. Proceedings of the Royal Society of London, Series B 277: 183-190.

Loewen M.A. 2009. Variation in the Late Jurassic theropod dinosaur Allosaurus: ontogenetic, functional, and taxonomic implications. Doctoral Thesis. The University of Utah.

\section{Lü J., Yi L., Brusatte S.L., Yang L., Li H., and Chen L. 2014. A new clade of Asian Late} Cretaceous long-snouted tyrannosaurids. Nature Communications 5: 3788

Madsen, J.H. Jr. 1976. Allosaurus fragilis: a revised osteology. Bulletin 109, Utah Geological Survey. $163 \mathrm{pp}$.

Maidment S.C.R., Bates K.T., Falkingham P.L., VanBuren C., Arbour V., and Barrett P.M. 2014. Locomotion in ornithischian dinosaurs: an assessment using three-dimensional computational modelling. Biological Reviews 89: 588-617.

Mallison, H. 2007. Virtual Dinosaurs - Developing Computer Aided Design and Computer Aided Engineering Modeling Methods for Vertebrate Paleontology. Doctoral Thesis. Eberhard-KarlsUniversität, Tübingen, Germany. http://tobias-lib.ub.uni-tuebin- gen.de/volltexte/2007/2868/

Mallison H. 2010. The digital Plateosaurus I: body mass, mass distribution and posture assessed using CAD and CAE on a digitally mounted complete skeleton. Palaeontologia Electronica 13(2) 8A: 26p.

Mallison H. 2014. Osteoderm distribution has low impact on the centre of mass of stegosaurs. Fossil Record 17:33-39.

Mallison H., Pittman M. and Schwarz D. 2015. Using crocodilian tails as models for dinosaur tails. PeerJ PrePrints https://dx.doi.org/10.7287/peerj.preprints.1339v1

Matthew W.D. and Brown B. 1923. Preliminary notices of skeletons and skulls of Deinodontidae from the Cretaceous of Alberta. American Museum Novitates 89: 1-10. 
1202 Mazzetta G.V., Christiansen P., and Fariña R.A. 2004. Giants and bizarres: body size of some 1203 southern South American Cretaceous dinosaurs. Historical Biology 2004: 1-13.

1204

1205

1206

1207

1208

1209

1210

1211

1212

1213

1214

1215

1216

1217

1218

1219

1220

1221

1222

1223

1224

1225

1226

1227

1228

1229

1230

1231

1232

1233

1234

1235

1236

1237

1238

1239

1240

1241

1242

1243

1244

1245

1246

McGowen A.J. and Dyke G.J. 2009. A surfeit of theropods in the Moroccan Late Cretaceous?

Comparing diversity estimates from field data and fossil shops. Geology 37: 843-846.

Moen D.S., Irschick D.J., and Wiens J.J. 2013. Evolutionary conservatism and convergence both lead to striking similarity in ecology, morphology and performance across continents in frogs. Proceedings of the Royal Society B 280: 20132156.

Moen D.S., Morlon H., and Wiens J.J. 2016. Testing convergence versus history: convergence dominates phenotypic evolution for over 150 million years in frogs. Systematic Biology 65: 146160 .

Motani R. 2001. Estimating body mass from silhouettes: testing the assumption of elliptical body cross-sections. Paleobiology 27: 735-750.

Orme D., Freckleton R., Thomas G., Petzoldt T., Fritz S., Isaac N., and Pearse W. 2013. caper: Comparative Analyses of Phylogenetics and Evolution in R. R package version 0.5.2. http://CRAN.R-project.org/package=caper

Osborn H.F. 1917. Skeletal adaptations of Ornitholestes, Struthiomimus, Tyrannosaurus. Bulletin of the American Museum of Natural History 35: 733-771.

Packard G.C., Boardman T.J., and Birchard G.F. 2009. Allometric equations for predicting body mass of dinosaurs. Journal of Zoology 279: 1-9.

Patel A. and M. Braae M. Rapid turning at high-speed: Inspirations from the cheetah's tail. 2013 IEEE/RSJ International Conference on Intelligent Robots and Systems, Tokyo. pp. 5506-5511.

Patel A., Boje E., Fisher C., Louis L., and Lane E. 2016. Quasi-steady state aerodynamics of the cheetah tail. Biology Open 15;5(8):1072-6.

Paul G.S. 2010. The Princeton Field Guide to Dinosaurs. Princeton University Press. Princeton.

Paul G.S. 1988. Predatory Dinosaurs of the World. A Complete Illustrated Guide. Simon \& Schuster. New York.

Peirce B.A.M. 1837. An Elementary Treatise on Plane and Solid Geometry. James Munroe and Company. Boston.

Persons W.S. IV and Currie P.J. 2011. The tail of Tyrannosaurus: reassessing the size and locomotive importance of the M. caudofemoralis in non-avian theropods. Anatomical Record 294: 119-131. 
1247 Peterson M.D., Alvar B.A., and Rhea M.R. 2006. The contribution of muscle force to explosive 1248 movement among young collegiate athletes. Journal of Strength and Conditioning Research 20: 1249 867-873.

1250

1251

1252

1253

1254

1255

1256

1257

1258

1259

1260

1261

1262

1263

1264

1265

1266

1267

1268

1269

1270

1271

1272

1273

1274

1275

1276

1277

1278

1279

1280

1281

1282

Rankin J.W., Rubenson J., and Hutchinson J.R. 2016 Inferring muscle functional roles of the ostrich pelvic limb during walking and running using computer optimization. Journal of the Royal Society Interface 13: 20160035.

Rayfield, E.J. 2004. Cranial mechanics and feeding in Tyrannosaurus rex. Proceedings of the Royal Society of London, Series B 271: 1451-1459.

Revell L.J. 2010. Phylogenetic signal and linear regression on species data. Methods in Ecology and Evolution 1: 319-329.

Rosin P.L. 2000. Fitting superellipses. IEEE Transactions on Pattern Analysis and Machine Intelligence 22: 726-732.

Russell D.A. 1970. Tyrannosaurs from the Late Cretaceous of western Canada. National Museum of Natural Sciences Publications in Paleontology 1: 1-34.

Russell D.A. and Paesler M.A. 2003. Environments of Mid-Cretaceous Saharan dinosaurs. Cretaceous Research 24: 569-588.

Sellke T., Bayarri M.J., and Berger J.O. 2001 Calibration of $p$ values for testing precise null hypotheses. The American Statistician 55: 62-71.

Sereno P.C., Tan L., Brusatte S.L., Kriegstein H.J., Zhao X., and Cloward K. 2009.

Tyrannosaurid skeletal design first evolved at small body size. Science. 326: 418-422.

Smaers J.B. 2014. evomap: R package for the evolutionary mapping of continuous traits. Available at Github: https://github.com/JeroenSmaers/evomap.

Smaers J.B. and Rohlf F.J. 2016. Testing species' deviation from allometric predictions using the phylogenetic regression. Evolution 70: 1145-1149.

Snively E. 2012. Rigid body mechanics of prey capture in large carnivorous dinosaurs. M.Sc.

1283

1284

1285

Thesis. Ohio University.

1286

Snively E. and Russell A.P. 2007a. Functional variation of neck muscles and their relation to

1287 feeding style in Tyrannosauridae and other large theropods. Anatomical Record 290: 934-957

1288 Snively E. and Russell A.P. 2007b. Craniocervical feeding dynamics of Tyrannosaurus rex.

1289 Paleobiology 33: 610-638.

1290

1291 Snively E. and Russell A.P. 2003. Kinematic model of tyrannosaurid (Dinosauria: Theropoda)

1292 arctometatarsus function. Journal of Morphology 255: 215-227. 
1293

1294

1295

1296

1297

1298

1299

1300

1301

1302

1303

1304

1305

1306

1307

1308

1309

1310

1311

1312

1313

1314

1315

1316

1317

1318

1319

1320

1321

1322

1323

1324

1325

1326

1327

1328

1329

1330

1331

1332

1333

1334

1335

1336

1337

Snively E., Henderson D.M., and Phillips D.S. 2006. Fused and vaulted nasals of tyrannosaurid dinosaurs: implications for cranial strength and feeding mechanics. Acta Palaeontia Polonica 51: 435-454.

Snively E., Henderson D.M., Wick E., Sokup R., Roth P., and Dupor M. 2015. Ceratopsian dinosaurs could turn more quickly and iguanodontians comparably to contemporaneous large theropods. Journal of Vertebrate Paleontology 35:216A.

Stoinski S., Suthau T., and Gunga H.C. 2011. Reconstructing body volume and surface area of dinosaurs using laser scanning and photogrammetry. In: Klein N., Remes K., Gee C.T. and Sander P.M. (eds.). Biology of the Sauropod Dinosaurs: Understanding the Life of Giants. Indiana University Press. Bloomington. pp. 94-104.

Sullivan, R.M. and Lucas, S.G. 2006. The Kirtlandian land-vertebrate "age" - faunal composition, temporal position and biostratigraphic correlation in the nonmarine Upper Cretaceous of western North America._New Mexico Museum of Natural History and Science, Bulletin 35:7-29.

Symonds M.R.E. and Blomberg S.P. 2014. A primer on phylogenetic generalised least squares (PGLS). In: Garamszegi L.Z. (ed.). Modern Phylogenetic Comparative Methods and Their Application in Evolutionary Biology: Concepts and Practice Springer. Berlin. pp 105-130.

Taylor M.P. 2009. A re-evaluation of Brachiosaurus altithorax Riggs 1903 (Dinosauria, Sauropoda) and its generic separation from Giraffatitan brancai (Janensch 1914). Journal of Vertebrate Paleontology 29: 787-806.

Therrien F. and Henderson D.M. 2007. My theropod is bigger than yours... or not: estimating body size from skull length in theropods. Journal of Vertebrate Paleontology 27: 108-115.

Thomas K., French D., and Hayes P.R. 2009. The effect of two plyometric training techniques on muscular power and agility in youth soccer players. Journal of Strength and Conditioning Research 23: 332-335.

Tinius A., Russell A.P., Jamniczky H.A., and Anderson J.S. 2018. What is bred in the bone: Ecomorphological associations of pelvic girdle form in greater Antillean Anolis lizards. Journal of Morphology 2018;00: 1-15.

Trinkaus E., Churchill S.E., Villemeur I., Riley K.G., Heller J.A., and Ruff C.B. 1991. Robusticity versus shape: the functional interpretation of Neandertal appendicular morphology. Journal of the Anthropological Society of Nippon 99: 257-278.

Varricchio D.J. 2001. Gut contents from a Cretaceous tyrannosaurid: implications for theropod dinosaur digestive tracts. Journal of Paleontology 75: 401-406. 
1338 Walker A.D. 1964. Triassic reptiles from the Elgin area: Ornithosuchus and the origin of

1339 carnosaurs. Philosophical Transactions of the Royal Society of London 248: 53-134.

1340

1341 Wegweiser M., Breithaupt B., and Chapman R. 2004. Attack behavior of tyrannosaurid

1342 dinosaur(s): Cretaceous crime scenes, really old evidence, and "smoking guns." Journal of

1343 Vertebrate Paleontology 24(Supplement 3), 127A.

1344

1345 Weiss T., Kreitinger J., Wilde H., Wiora C., Steege M., Dalleck L., and Janot J. 2010. Effect of

1346 functional resistance training on muscular fitness outcomes in young adults. Journal of Exercise

1347 Science and Fitness 2: 113-122.

1348

1349 Wilson A.M., Lowe J.C., Roskilly K., Hudson P.E., Golabek K.A., and McNutt J.W. 2013.

1350

1351

1352

1353

Locomotion dynamics of hunting in wild cheetahs. Nature 498: 185-189.

Witmer L.M. and Ridgely R.C. 2008. The paranasal air sinuses of predatory and armored dinosaurs (Archosauria: Theropoda and Ankylosauria) and their contribution to cephalic

1354

1355

1356

1357 structure. Anatomical Record 291: 1362-1388.

Young W.B., James R., and Montgomery I. 2002. Is muscle power related to running speed with changes in direction? Journal of Sports Medicine and Physical Fitness 42: 282-288. 
1359 Table 1. Muscles originating from the ilium and tail of theropod dinosaurs (Carrano and 1360 Hutchinson 2002, Mallison et al. 2015) and their utility for yaw (turning the body laterally).

1361 Although few muscles pivot the body directly over the stance leg (Mm. caudofemoralis brevis et 1362 longus, M. ilio-ischiocaudalis), all large ilium-based muscles are potentially involved with 1363 turning by acceleration of the body on the outside of the turn, stabilization of the hip joint, or 1364 conservation of angular momentum by swinging the tail.

\begin{tabular}{|c|c|c|}
\hline Muscle & Action & Effect on turning (yaw) \\
\hline \multicolumn{3}{|l|}{ Ilium origin } \\
\hline M. iliotibialis 1 & Knee extension, hip flexion & $\begin{array}{l}\text { Greater acceleration outside turn, } \\
\text { stabilization inside turn }\end{array}$ \\
\hline M. iliotibialis 2 & Knee extension, hip flexion & $\begin{array}{l}\text { Greater acceleration outside turn, } \\
\text { stabilization inside turn }\end{array}$ \\
\hline M. iliotibialis 3 & Knee extension & $\begin{array}{l}\text { Greater acceleration outside turn, } \\
\text { stabilization inside turn }\end{array}$ \\
\hline M. iliotrochantericus caudalis & Hip abduction & Joint stabilization \\
\hline M. iliofemoralis externus & Hip abduction & Joint stabilization \\
\hline M. iliofemoralis internus & Hip abduction & Joint stabilization \\
\hline M. caudofemorais brevis & $\begin{array}{l}\text { Femoral retraction, direct yaw of } \\
\text { body, pitch of body }\end{array}$ & $\begin{array}{l}\text { Yaw with unilateral contraction, } \\
\text { contralateral braking }\end{array}$ \\
\hline \multicolumn{3}{|l|}{ Tail origin } \\
\hline M. caudofemoralis longus & $\begin{array}{l}\text { Femoral retraction, direct yaw of } \\
\text { body, pitch of body }\end{array}$ & $\begin{array}{l}\text { Yaw with unilateral contraction, } \\
\text { Ipsilateral yaw by conservation of } \\
\text { angular momentum, contralateral } \\
\text { braking }\end{array}$ \\
\hline \multicolumn{3}{|l|}{ Ilium origin, tail insertion } \\
\hline M. ilio-ischiocaudalis (dorsal) & Tail lateral and dorsal flexion & $\begin{array}{l}\text { Ipsilateral yaw by conservation of } \\
\text { angular momentum, contralateral } \\
\text { braking }\end{array}$ \\
\hline
\end{tabular}


1366 Table 2. Theropod taxa, specimens, and data sources for calculations of mass, mass moment of 1367 inertia, and ilium area.

1368

\begin{tabular}{|c|c|c|c|c|}
\hline Taxon & Specimen \# & Lateral view & $\begin{array}{l}\text { Dorsal view/ } \\
\text { modified from }\end{array}$ & Ilium source \\
\hline Dilophosaurus wetherelli & UCMP 37302 & $\begin{array}{l}\text { Paul 2010, } \\
\text { Hartman } 2015, \\
\text { Allen et al. } 2013\end{array}$ & $\begin{array}{l}\text { Paul } 2010 \dagger, \text { Allen et } \\
\text { al. } 2013\end{array}$ & Hartman 2015 \\
\hline Ceratosaurus nasicornis & USNM 4735 & Paul 2010 & Paul 2010 & $\begin{array}{l}\text { photo; Gilmore } \\
1920\end{array}$ \\
\hline \multicolumn{5}{|l|}{ Basal tetanurae } \\
\hline $\begin{array}{l}\text { Eustreptospondylus } \\
\text { oxoniensis }\end{array}$ & OUM J13558 & Paul 2010 & $\begin{array}{l}\text { Paul 1988, Walker } \\
1964\end{array}$ & Walker 1964 \\
\hline Allosaurus fragilis & $\begin{array}{l}\text { USNM } 4734, \\
\text { UUVP } 6000\end{array}$ & $\begin{array}{l}\text { Paul 2010, Paul } \\
1988\end{array}$ & Paul 2010 & $\begin{array}{l}\text { Paul 2010, } \\
\text { Madsen } 1976\end{array}$ \\
\hline $\begin{array}{l}\text { Allosaurus jimmadseni } \\
\text { (tail restored) }\end{array}$ & MOR 693 & Bates et al. 2009 & Paul 2010 & $\begin{array}{l}\text { photo; Loewen } \\
2009\end{array}$ \\
\hline Acrocanthosaurus atokensis & NCSM 14345 & Bates et al. 2010 & Bates et al. 2010 & $\begin{array}{l}\text { photo, Bates et } \\
\text { al. } 2012 \\
\text { (restored) }\end{array}$ \\
\hline Giganotosaurus carolinii & MUCPv-CH-1 & $\begin{array}{l}\text { Paul 2010, } \\
\text { Hartman } 2015\end{array}$ & $\begin{array}{l}\text { Paul 2010, Coria and } \\
\text { Currie } 2002 \dagger\end{array}$ & $\begin{array}{l}\text { photo; Hartman } \\
2015\end{array}$ \\
\hline Sinraptor hepingensis & ZDM 0024 & Paul 2010 & Paul 2010, Gao 1992 & Gao 1992 \\
\hline $\begin{array}{l}\text { Yangchuanosaurus } \\
\text { shangyouensis }\end{array}$ & CV 00215 & Paul 2010 & Paul 2010 & Dong et al. 1983 \\
\hline \multicolumn{5}{|l|}{ Tyrannosauroidea } \\
\hline $\begin{array}{l}\text { Raptorex kriegsteini (small } \\
\text { juvenile Tarbosaurus) }\end{array}$ & LH PV18 & Paul 2010 & Sereno et al. 2010 & $\begin{array}{l}\text { Sereno et al. } \\
2010\end{array}$ \\
\hline Tarbosaurus bataar (juvenile) & ZPAL MgD-I/3 & Paul 1988, 2010 & Paul $1988 \dagger$ & photo; Paul 1988 \\
\hline Tarbosaurus bataar (adult) & ZPAL MgD-I/4 & Paul 2010 & $\begin{array}{l}\text { Hurum and Sabath } \\
2003\end{array}$ & photo \\
\hline Tarbosaurus bataar (adult) & PIN 552-1 & Paul 2010 & Paul 1988† & $\begin{array}{l}\text { Paul 1988, } \\
\text { Maleev } 1974\end{array}$ \\
\hline Tyrannosaurus rex (juvenile) & BMRP 2002.4.1 & Paul 2010 & $\begin{array}{l}\text { Persons and Currie } \\
2011\end{array}$ & photo; Paul 2010 \\
\hline Tyrannosaurus rex (adult) & $\begin{array}{l}\text { AMNH 5027, CM } \\
9380\end{array}$ & $\begin{array}{l}\text { Paul 2010, } \\
\text { Hartman } 2004\end{array}$ & $\begin{array}{l}\text { Persons and Currie } \\
2011\end{array}$ & $\begin{array}{l}\text { photo; Osborn } \\
1917\end{array}$ \\
\hline Tyrannosaurus rex (adult) & FMNH PR 2081 & Hartman 2004 & $\begin{array}{l}\text { Persons and Currie } \\
2011\end{array}$ & $\begin{array}{l}\text { photo; Brochu } \\
2003\end{array}$ \\
\hline Gorgosaurus libratus (adult) & $\begin{array}{l}\text { AMNH 5458, NMC } \\
2120\end{array}$ & Paul 2010, 1988 & Paul 1988 & photo; Paul 2010 \\
\hline $\begin{array}{l}\text { Gorgosaurus libratus } \\
\text { (juvenile) }\end{array}$ & AMNH 5664 & Paul 2010 & Paul 1988 & $\begin{array}{l}\text { photo; Matthew } \\
\text { and Brown } 1923\end{array}$ \\
\hline $\begin{array}{l}\text { Gorgosaurus libratus } \\
\text { (juvenile) }\end{array}$ & TMP 91.36.500 & $\begin{array}{l}\text { Currie } 2003, \\
\text { Hartman } 2015\end{array}$ & Paul 1988 & $\begin{array}{l}\text { photo; Currie } \\
2003 \text {, Hartman } \\
2015\end{array}$ \\
\hline Daspletosaurus torosus & CMN 8506 & Paul 2010 & $\begin{array}{l}\text { Paul 1988, Russell } \\
1970\end{array}$ & Russell 1970 \\
\hline
\end{tabular}

$\dagger=$ Different genus used for modified dorsal body outline.

1371 Institutional abbreviations: $\mathrm{AMNH}=\mathrm{American}$ Museum of Natural History. BMRP=Burpee Museum (Rockford),

1372 Paleontology. $\mathrm{CM}=$ Carnegie Museum of Natural History. $\mathrm{CMN}=$ Canadian Museum of Nature. $\mathrm{CV}=$ Municipal

1373 Museum of Chunking. FMNH=Field Museum of Natural History. LH PV=Long Hao Institute of Geology and 
1374 Paleontology. MOR=Museum of the Rockies. MUCPv=Museo de la Universidad Nacional del Comahue, El Chocón 1375 collection. NCSM=North Carolina State Museum. NMC= National Museum of Canada. OUM=Oxford University

1376 Museum. PIN=Paleontological Institute, Russian Academy of Sciences. TMP=Royal Tyrrell Museum of

1377 Palaeontology; UCMP=University of California Museum of Paleontology. USNM= United States National Museum.

1378 UUVP=University of Utah Vertebrate Paleontology. ZDM= Zigong Dinosaur Museum. ZPAL=Paleobiological

1379 Institute of the Polish Academy of Sciences.

1380

1381 
1382 Table 3. Ilium area, mass properties, and relative agility of theropod dinosaurs. Mass properties

1383 are "best estimate" values, assuming superellipse body cross sections with exponent $\mathrm{k}=2.3$

1384 (compared with $\mathrm{k}=2$ for an ellipse). This cross section is common for terrestrial vertebrates, and

1385 has $4.7 \%$ greater area than an ellipse of the same radii. Differing exponents, specific tension

1386 coefficients for absolute muscle force, and relative moment arms (scaled as body mass ${ }^{1 / 3}$ ) do not

1387 change relative agilities of tyrannosaurids and large non-tyrannosaurids predatory theropods.

1388 Agility $_{\text {force }}$ is an estimate of relative maneuverability based on a human athletic standard that

1389 finds turning ability is highly correlated with leg muscle force/body mass ratio. Agility moment $_{\text {t }}$

1390 enables comparison of turning ability by incorporating scaled moment arms for estimating

1391 relative torques. As a first approximation, Agility $y_{\text {moment }}$ assumes similar scaling of moment arms

1392 across all taxa. 


\begin{tabular}{|c|c|c|c|c|c|c|c|c|c|c|}
\hline & \multirow{2}{*}{$\begin{array}{l}\begin{array}{c}\text { Ilium } \\
\text { area }\end{array} \\
\mathrm{A}\left(\mathrm{cm}^{2}\right)\end{array}$} & \multicolumn{2}{|c|}{ Total Mass } & \multicolumn{3}{|c|}{ Mass moments of inertia } & \multirow{2}{*}{$\begin{array}{c}\begin{array}{c}\text { Agility } \\
\text { force } \\
\text { axial body }\end{array} \\
\text { A/I }\end{array}$} & \multirow{2}{*}{$\begin{array}{c}\begin{array}{c}\text { Agility } \\
\text { moment } \\
\text { axial body }\end{array} \\
\tau_{\text {relative }} / \mathrm{I}\end{array}$} & \multirow{2}{*}{$\begin{array}{c}\begin{array}{c}\text { Agility } \\
\text { force } \\
\text { body+leg }\end{array} \\
\text { A/I }\end{array}$} & \multirow{2}{*}{ 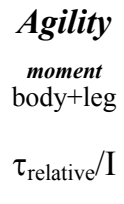 } \\
\hline & & $\mathrm{kg}$ & $\log 10$ & $\begin{array}{c}I_{y b o d y} \\
\left(\operatorname{kgxm}^{2}\right)\end{array}$ & $\begin{array}{c}I_{y \text { leg }} \\
\left(\mathrm{kgxm}^{2}\right)\end{array}$ & $\begin{array}{l}\mathrm{I}_{\mathrm{y} \text { body+leg }} \\
\left(\mathrm{kgxm}^{2}\right)\end{array}$ & & & & \\
\hline Taxon & & & & & & & & & & \\
\hline Dilophosaurus wetherelli & 380.16 & 372.07 & 2.571 & 213 & 0.279 & 218 & 1.78 & 2.57 & 1.75 & 2.51 \\
\hline Ceratosaurus nasicornis & 903.83 & 678.26 & 2.831 & 546 & 1.093 & 559 & 1.60 & 2.21891 & 1.57 & 2.61 \\
\hline $\begin{array}{l}\text { Eustreptospondylus } \\
\text { oxoniensis }\end{array}$ & 280 & 206.26 & 2.314 & 70.45 & 0.098 & 73.26 & 3.97 & 4.70 & 3.82 & 4.52 \\
\hline Allosaurus fragilis & 1131.5 & 1512.10 & 3.180 & 2303.25 & 2.405 & 2344.62 & 0.49 & 1.13 & 0.48 & 1.11 \\
\hline Allosaurus fragilis & 1228.06 & 1683.33 & 3.226 & 2036.81 & 2.121 & 2078.55 & 0.60 & 1.43 & 0.59 & 1.41 \\
\hline $\begin{array}{l}\text { Acrocanthosaurus } \\
\text { atokensis }\end{array}$ & 2551.25 & 5474.1 & 3.738 & 14979 & 19.718 & 15377.24 & 0.17 & 0.60 & 0.17 & 0.58 \\
\hline Giganotosaurus carolinii & 3540.64 & 6907.6 & 3.839 & 35821 & 23.731 & 26593.36 & 0.10 & 0.511 & 0.13 & 0.507 \\
\hline Sinraptor hepingensis & 1268.9 & 2373.5 & 3.430 & 3530.7 & 4.929 & 3740.32 & 0.343 & 0.93 & 0.339 & 0.91 \\
\hline $\begin{array}{l}\text { Yangchuanosaurus } \\
\text { shangyouensis }\end{array}$ & 992.4 & 2176.4 & 3.173 & 2836.7 & 3.365 & 1672.88 & 0.61 & 1.36 & 0.59 & 1.31 \\
\hline Raptorex kriegsteini & 179.7 & 47.07 & 1.673 & 4.65 & 0.0205 & 4.68 & 43.96 & 31.74 & 43.60 & 31.49 \\
\hline $\begin{array}{l}\text { Tarbosaurus bataar } \\
\text { (juvenile) }\end{array}$ & 1455.2 & 727.45 & 2.861 & 535 & 1.437 & 548 & 2.72 & 2.39 & 2.65 & 4.77 \\
\hline $\begin{array}{l}\text { Tarbosaurus bataar } \\
\text { (adult) }\end{array}$ & 2800 & 2249.1 & 3.352 & 3069.9 & 5.586 & 3126.17 & 0.912 & 2.39 & 0.905 & 2.37 \\
\hline $\begin{array}{l}\text { Tarbosaurus bataar } \\
\text { (adult) }\end{array}$ & 2977 & 2816.3 & 3.450 & 4486 & 10.049 & 4515.1 & 0.664 & 1.87 & 0.659 & 1.86 \\
\hline $\begin{array}{l}\text { Tyrannosaurus rex } \\
\text { (juvenile) }\end{array}$ & 1107.41 & 660.23 & 2.820 & 344.83 & 0.683 & 347 & 3.21 & 5.59 & 3.19 & 5.56 \\
\hline Tyrannosaurus rex (adult) & 4786.49 & 6986.6 & 3.844 & 18175 & 34.067 & 18276.08 & 0.263 & 1.01 & 0.262 & 1.00 \\
\hline Tyrannosaurus rex (adult) & 6661.8 & 9130.87 & 3.963 & 28847 & 51.205 & 29297 & 0.231 & 0.97 & 0.227 & 0.95 \\
\hline $\begin{array}{l}\text { Gorgosaurus libratus } \\
\text { (adult) }\end{array}$ & 2358 & 2427.3 & 3.385 & 3219 & 9.79 & 3312 & 0.73 & 1.97 & 0.70 & 1.67 \\
\hline $\begin{array}{l}\text { Gorgosaurus libratus } \\
\text { (juvenile) }\end{array}$ & 1040.56 & 687.7 & 2.837 & 402 & 1.087 & 420.14 & 2.59 & 4.56 & 2.48 & 4.37 \\
\hline $\begin{array}{l}\text { Gorgosaurus libratus } \\
\text { (juvenile) }\end{array}$ & 1060.93 & 496.1 & 2.70 & 251.95 & 0.660 & 265.29 & 4.21 & 6.67 & 4.00 & 6.33 \\
\hline Daspletosaurus torosus & 3209.77 & 3084.8 & 3.489 & 5338 & 9.665 & 5586 & 0.60 & 1.75 & 0.58 & 1.67 \\
\hline
\end{tabular}


1398 Table 4. Centers of mass (COM) and rotation axes for large theropod dinosaurs. Axial body: The $1399 \mathrm{x}$ value is the position $(\mathrm{m})$ from the anterior tip of the rostrum (where $\mathrm{x}=0$ ), and $\mathrm{y}$ value is the 1400 distance $(\mathrm{m})$ from the ventral point of the body $(\mathrm{y}=0)$. The $\mathrm{z}$ position is 0 , at the midline of the 1401 body, because the body is modeled as symmetrical. Swing leg: This is the positive $\mathrm{z}$ coordinate 1402 position (in $\mathrm{m}$ ) of the leg relative to that of the axial body's COM. Axial body+swing leg: The $\mathrm{z}$ 1403 coordinate positon $(\mathrm{m})$ of the collective COM of the body and swing leg. The value is small 1404 because the leg's mass is much less than that of the axial body.

\begin{tabular}{|c|c|c|c|c|c|c|}
\hline \multirow[b]{2}{*}{ Taxon } & \multicolumn{2}{|c|}{ Axial body $\operatorname{COM}(\mathrm{z}=0)$} & \multicolumn{2}{|c|}{ Swing leg rotation axis } & \multicolumn{2}{|c|}{$\begin{array}{l}\text { Axial body }+ \text { swing leg } \\
\text { rotation axis }\end{array}$} \\
\hline & $\mathrm{x}$ & $\mathrm{y}$ & $\mathrm{x}$ & $\mathrm{Z}$ & $\mathrm{x}$ & $\mathrm{Z}$ \\
\hline Dilophosaurus wetherelli & 2.33 & 0.42 & 2.61 & 0.17 & 2.36 & 0.02 \\
\hline Ceratosaurus nasicornis & 2.66 & 0.50 & 3.07 & 0.15 & 2.70 & 0.01 \\
\hline $\begin{array}{l}\text { Eustreptospondylus } \\
\text { oxoniensis }\end{array}$ & 1.46 & 0.33 & 1.84 & 0.10 & 1.51 & 0.01 \\
\hline Allosaurus fragilis & 2.72 & 0.64 & 3.26 & 0.0 .24 & 2.77 & 0.02 \\
\hline Allosaurus jimmadseni & 2.64 & 0.79 & 3.20 & 0.23 & 2.69 & 0.02 \\
\hline Acrocanthosaurus atokensis & 4.34 & 0.91 & 4.69 & 0.46 & 4.36 & 0.03 \\
\hline Giganotosaurus carolinii & 4.54 & 1.33 & 5.10 & 0.44 & 4.57 & 0.03 \\
\hline Sinraptor hepingensis & 3.12 & 0.86 & 3.57 & 0.15 & 3.16 & 0.01 \\
\hline $\begin{array}{l}\text { Yangchuanosaurus } \\
\text { shangyouensis }\end{array}$ & 2.40 & 0.72 & 2.99 & 0.23 & 2.45 & 0.02 \\
\hline $\begin{array}{l}\text { Tarbosaurus bataar } \\
\text { (juvenile)/Raptorex }\end{array}$ & 0.87 & 0.22 & 0.05 & 0.0073 & 0.88 & 0.007 \\
\hline $\begin{array}{l}\text { Tarbosaurus bataar } \\
\text { (juvenile) }\end{array}$ & 1.93 & 0.54 & 2.33 & 0.15 & 1.98 & 0.02 \\
\hline Tarbosaurus bataar (ZPAL) & 2.85 & 0.80 & 0.31 & 0.027 & 2.87 & 0.014 \\
\hline Tarbosaurus bataar (adult) & 3.01 & 0.87 & 0.29 & 0.028 & 2.07 & 0.025 \\
\hline Tyrannosaurus rex (juvenile) & 2.19 & 0.60 & 0.16 & 0.018 & 2.19 & 0.02 \\
\hline Tyrannosaurus rex (adult) & 3.82 & 1.15 & 0.36 & 0.032 & 3.87 & 0.04 \\
\hline Tyrannosaurus rex (adult) & 3.84 & 1.17 & 0.40 & 0.040 & 3.90 & 0.04 \\
\hline Gorgosaurus libratus (adult) & 3.20 & 0.89 & 3.72 & 0.29 & 3.27 & 0.04 \\
\hline $\begin{array}{l}\text { Gorgosaurus libratus } \\
\text { (AMNH juvenile) }\end{array}$ & 1.73 & 0.49 & 2.21 & 0.18 & 1.79 & 0.02 \\
\hline $\begin{array}{l}\text { Gorgosaurus libratus (TMP } \\
\text { juvenile) }\end{array}$ & 2.03 & 0.52 & 2.51 & 0.13 & 2.10 & 0.02 \\
\hline Daspletosaurus torosus & 3.35 & 1.16 & 3.93 & 0.25 & 3.43 & 0.05 \\
\hline
\end{tabular}


1405 Table 5. Variation of mass properties with different tail widths. The last three columns are percentages relative to the baseline values. 1406

\begin{tabular}{|c|c|c|c|c|c|c|c|c|c|c|}
\hline Taxon & Specimen & $\begin{array}{c}\text { mass: initial } \\
\text { kg }\end{array}$ & $\begin{array}{c}\text { mass: } 1.4 \text { tail } \\
\text { kg }\end{array}$ & $\begin{array}{c}\text { CM initial } \\
\text { m from rostrum }\end{array}$ & $\begin{array}{c}\text { CM } \\
1.4 \text { tail }\end{array}$ & $\begin{array}{c}\quad I_{y} \\
\text { initial }\end{array}$ & $\begin{array}{c}I_{y} \\
1.4 \text { tail }\end{array}$ & $\begin{array}{c}\text { mass: } \\
\% \\
\text { initial }\end{array}$ & $\begin{array}{c}\text { CM: } \\
\% \\
\text { initial }\end{array}$ & $\begin{array}{c}I_{y}: \\
\% \\
\text { initial }\end{array}$ \\
\hline Tarbosaurus bataar & ZPAL MgD-I/4 & 2249 & 2367 & 2.68 & 2.97 & 3070 & 3578 & 105.2 & 110.8 & 116.5 \\
\hline Tyrannosaurus rex & AMNH 5027 & 6986 & 7458 & 3.82 & 4.01 & 18175 & 21395 & 106.7 & 105 & 117.7 \\
\hline Tyrannosaurus rex & FMNH PR 2081 & 9131 & 9657 & 3.79 & 4.24 & 28847 & 34742 & 105.1 & 111.9 & 120.4 \\
\hline Acrocanthosaurus atokensis & NCSM 14345 & 5603 & 6560 & 4.09 & 4.49 & 14978 & 22083 & 117.1 & 109.8 & 147.4 \\
\hline Allosaurus fragilis & USNM 4734 & 1356 & 1456 & 2.42 & 2.78 & 1662 & 1982 & 107.4 & 114.9 & 119.3 \\
\hline Yanchuanosaurus shangyouensis & CV 00215 & 1362 & 1441 & 2.64 & 2.95 & 1613 & 1905 & 105.8 & 111.7 & 118.1 \\
\hline Sinraptor hepingensis & ZDM 0024 & 2428 & 2588 & 3.12 & 3.37 & 3694 & 4374 & 106.6 & 108 & 118.4 \\
\hline
\end{tabular}


1408 Table 6. Regression statistics and comparisons of Agility $_{\text {force }}$ and Agility $_{\text {moment }}$ between groups of theropods turning their bodies, with 1409 both legs planted on the ground. Among groups compares all three groups together. Tyrannosaurs vs. Juveniles compares adult and 1410 juvenile tyrannosaurid specimens, and All Tyrannosaurs vs. Other Theropods combines juvenile and adult tyrannosaurids.

1411 Tyrannosaurids have significantly greater agility values than other theropods regardless of grouping, but juvenile and adult 1412 tyrannosaurids share an allometric continuum.

\section{AGILITY PLANTED}

\section{Regression Statistics}

$\begin{array}{lcc} & \begin{array}{c}\text { Agility } \\ \text { Force }\end{array} & \begin{array}{c}\text { Agility } \\ \text { Moment }\end{array} \\ \text { Multiple R } & 0.861 & 0.939 \\ \text { Adjusted R } & & \\ \text { Residual Standard } & 0.853 & 0.935 \\ \text { Error } & 0.0296 & 0.0197 \\ \text { Degrees of Freedom } & 18 & 18 \\ \text { F-Statistic } & 111.3 & 275.6 \\ \text { Slope } & -1.01 & -0.708 \\ \text { Intercept } & 2.92 & 2.246 \\ \mathrm{p} & 3.9 \times 10^{-9} & \end{array}$

\section{Agility Force \\ ANCOVA}

Among Groups
Juvenile vs. Adult
Tyrannosaurs
All Tyrannosaurs
vs. Theropods

F

$\mathrm{P}$

$a(p)$

$\begin{array}{lll}10.2 & 0.0014 & 0.0244\end{array}$

0.0054

0.9421

0.1325

19.45

0.0004

\section{Agility Moment \\ ANCOVA}

$\begin{array}{cccc} & \mathrm{F} & \mathrm{P} & a(p) \\ \begin{array}{ccc}\text { Among Groups } \\ \text { Juvenile vs. Adult }\end{array} & 6.71 & 0.077 & 0.3492 \\ \begin{array}{c}\text { Tyrannosaurs } \\ \text { All Tyrannosaurs }\end{array} & 0.008 & 0.9301 & 0.1548 \\ \begin{array}{c}\text { vs. Theropods } \\ \text { (1) }\end{array} & 12.91 & 0.0024 & 0.0379\end{array}$


1415 Table 7. Regression statistics and comparisons of Agility $_{\text {force }}$ and Agility $_{\text {moment }}$ between groups of theropods turning while pivoting on

1416 one foot ("en pointe"). Among groups compares all three groups together. Tyrannosaurs vs. Juveniles compares adult and juvenile

1417 tyrannosaurid specimens, and All Tyrannosaurs vs. Other Theropods combines juvenile and adult tyrannosaurids. Tyrannosaurids

1418 have significantly greater agility values than other theropods regardless of grouping, but juvenile and adult tyrannosaurids share an

1419 allometric continuum.

\section{AGILITY EN}

POINTE

\section{Regression}

Statistics

$\begin{array}{lcc} & \begin{array}{c}\text { Agility } \\ \text { Force }\end{array} & \begin{array}{c}\text { Agility } \\ \text { Moment } \\ \text { Multiple R^2 }\end{array} \\ & 0.862 & 0.941 \\ \text { Adjusted R^2 } & 0.853 & 0.9373 \\ \text { Residual Standard } & 0.8543 & 0.0192 \\ \text { Error } & & \\ \text { Degrees of } & 18 & 18 \\ \text { Freedom } & 112.4 & 284.9 \\ \text { F-Statistic } & -1.02 & -0.706 \\ \text { Slope } & 2.92 & 2.23 \\ \text { Intercept } & 3.61 \times 10^{-9} & 1.73 \times 10^{-12} \\ \text { p } & & \end{array}$

Agility Force ANCOVA

Among Groups
Juvenile vs. Adult
Tyrannosaus
All Tyrannosaurs

vs. Theropods

\section{Agility Moment ANCOVA}

$\begin{array}{ccc}\mathrm{F} & \mathrm{P} & a(p) \\ 10.44 & 0.0013 & 0.0229 \\ 0.149 & 0.7044 & 0.4015 \\ 19.21 & 0.0005 & 0.0102\end{array}$

Among Groups
Juvenile vs. Adult
Tyrannosaus
All Tyrannosaurs
vs. Theropods

$\begin{array}{ccc}\mathrm{F} & \mathrm{P} & a(p) \\ 10.69 & 0.0011 & 0.0200 \\ 0.160 & 0.6938 & 0.4081 \\ 19.61 & 0.0004 & 0.0084\end{array}$


1421

1422

1423
1424

1425

1426

1427

1428

1429

1430

1431

1432

1433

1434
Figure 1.

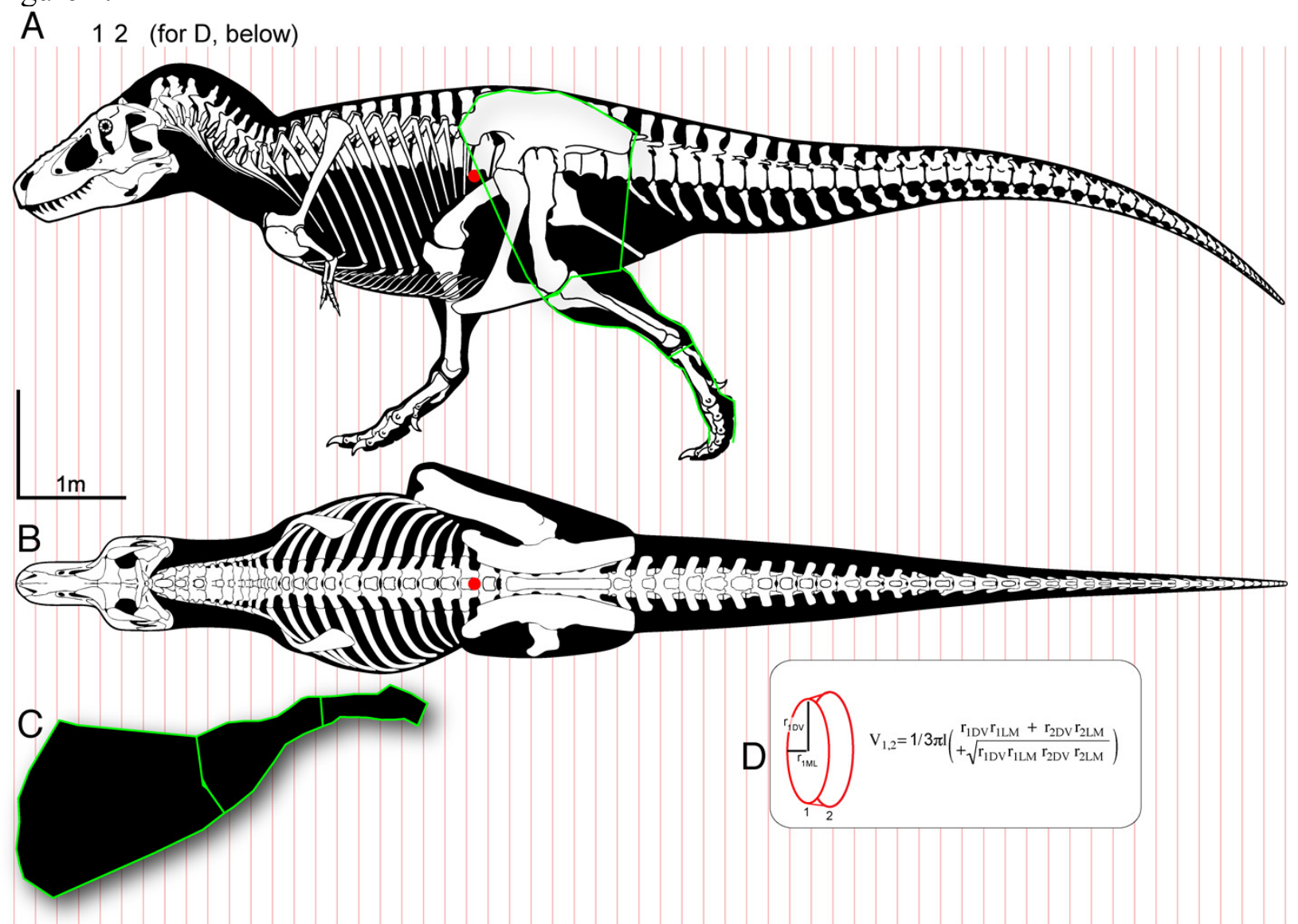

Figure 1. Methods for digitizing body outlines and calculating mass properties, for "maximum tail width" estimate for Tyrannosaurus rex. Reconstructions of Tyrannosaurus rex (Field Museum FMNH PR 2081) in lateral view (A) and dorsal view (B) enable digitizing of dorsal, ventral, and lateral extrema where they cross the vertical red lines. The lateral view (A) is modified with the dorsal margin of the neck conservatively raised based on recent muscle reconstructions (Snively and Russell 2007a, b). The hind leg (A and C) is outlined in green, and straightened (C) for digitizing. A red dot (A and B) specifies the center of mass of the axial body (minus the limbs) using this reconstruction. An equation for the volume of a given frustum of the body (D), between positions 1 and 2, assumes elliptical cross sections. 
1435

1436

1437

1438

1439

1440

1441
1442

1443

1444

1445

1446

1447

1448

1449

1450

1451

1452

1453

1454

1455

1456

1457

Figure 2.

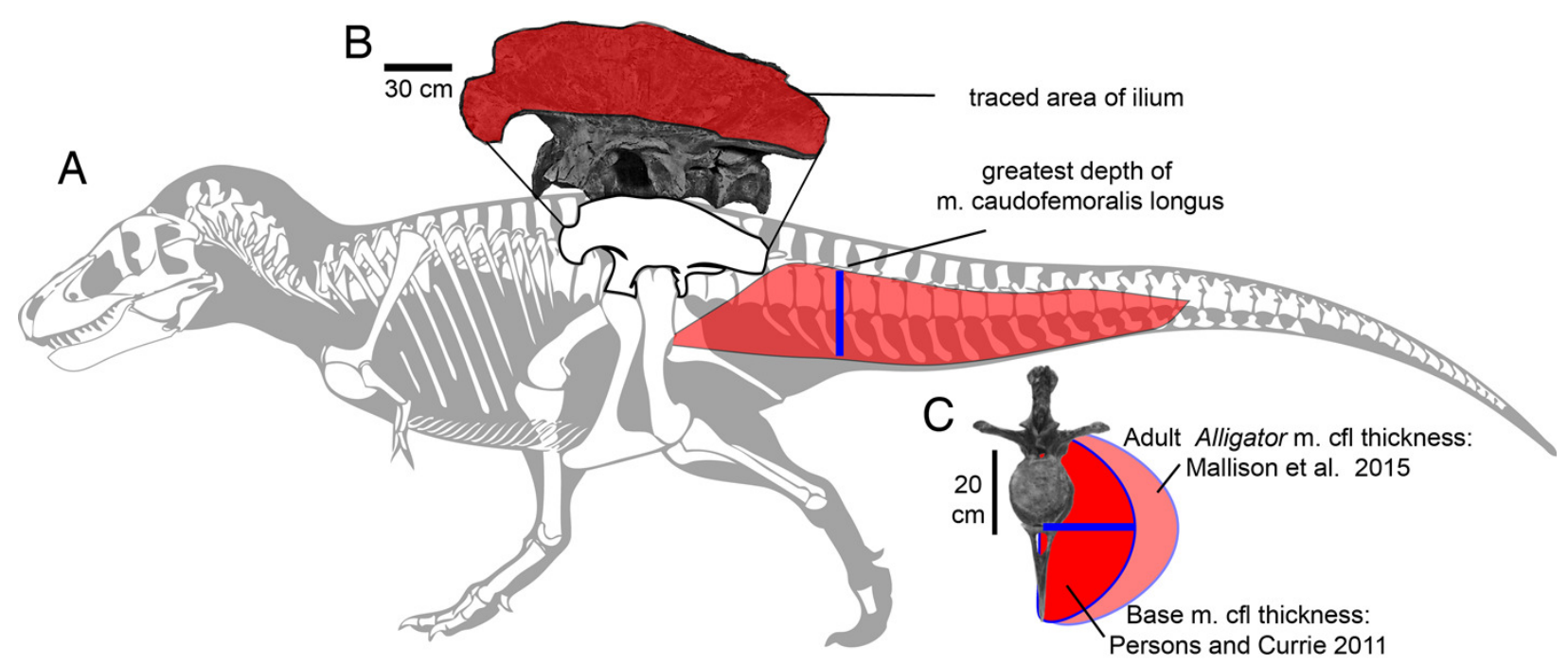

Figure 2. Methods for approximating attachment cross-sectional area of hind limb muscles, on lateral view (A) of a Tyrannosaurus rex skeleton (FMNH PR 2081; modified from Hartman 2011). The blue line shows the position of the greatest depth from the caudal ribs to the ventral tips of the chevrons, and greatest inferred width of the m. caudofemoralis longus. B. The inferred region of muscle attachment on the ilium (modified from Brochu 2003) is outlined in red, for scaled area measurement in ImageJ. C. The initial reconstructed radius (blue) of $\mathrm{m}$. caufofemoralis longus (CFL) is 0.5 times the hypaxial depth of the tail (blue line in a), seen in anterior view of free caudal vertebra 3 and chevron 3. The maximum lateral extent of CFL is here based on cross-sections of adult Alligator mississippiensis (Mallison et al. 2015). Note that the chevron in $\mathrm{c}$ is modified to be 0.93 of its full length, because it slopes posteroventrally when properly articulated (Brochu 2003). Bone images in A and C are "cartoonized" in Adobe Photoshop to enhance edges. 
1458 Figure 3 (caption on next page).

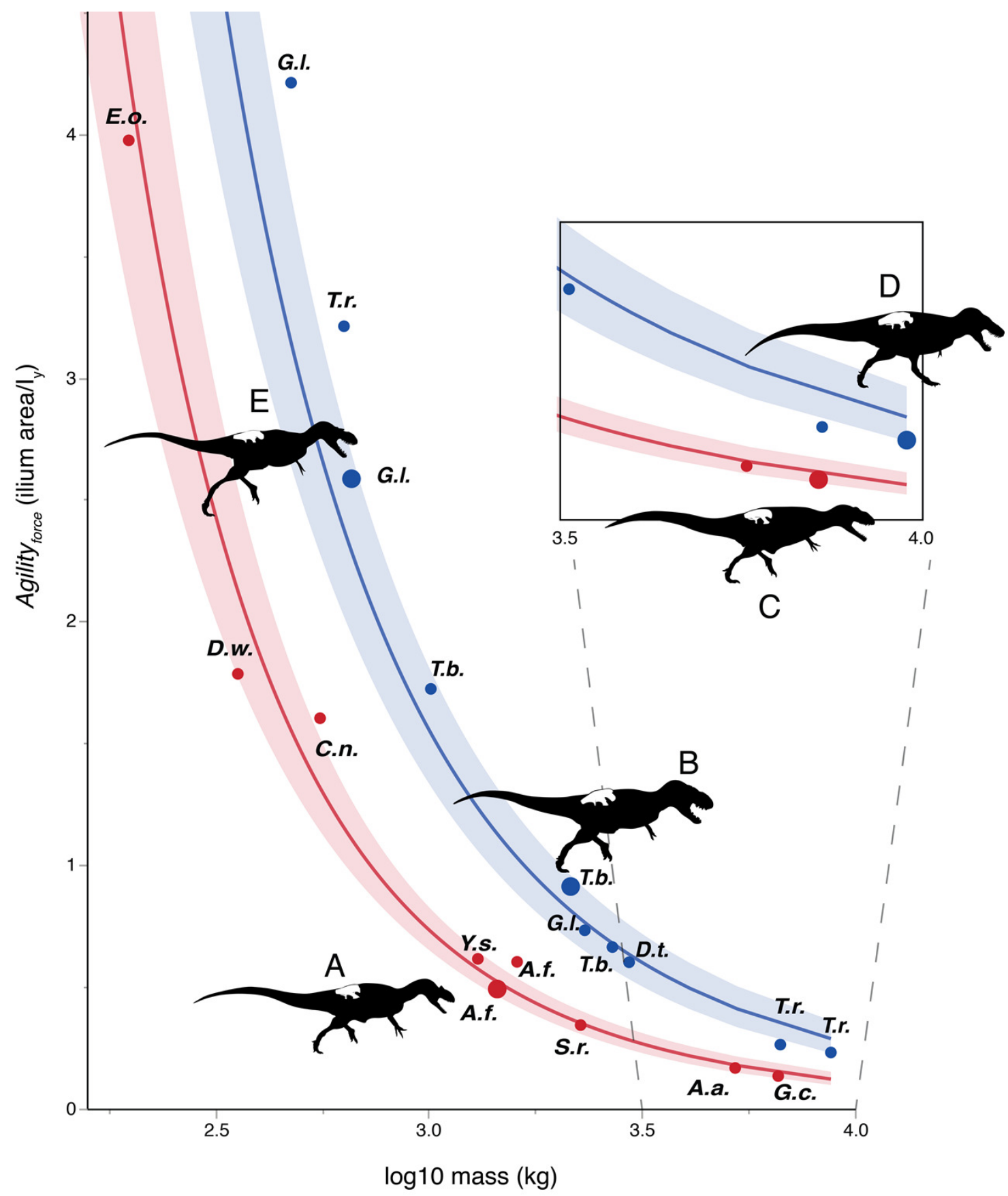


1466 Figure 3. Log-linear plot of body mass (x-axis) versus an agility index (y-axis) based on muscles

1467 originating from the ilium, with tyrannosauruids in blue and non-tyrannosaurids in red. 95\%

1468 confidence intervals do not overlap. Larger circles show positions of depicted specimens. A.

1469 Allosaurus fragilis. B. Tarbosaurus bataar. C. Giganotosaurus carolinii (a shorter-headed

1470 reconstruction was used for regressions). D. Tyrannosaurus rex. E. Gorgosaurus libratus

1471 (juvenile). The Tyrannosaurus rex silhouette is modified after Hartman (2011); others are

1472 modified after Paul (1988, 2010). The inset enlarges results for theropods larger than 3 tonnes in

1473 mass. Note that the tyrannosaurids have 2-5 times the agility index magnitudes of other

1474 theropods of similar mass. Discrepancies between tyrannosaurids and non-tyrannosaurids are

1475 greater at smaller body sizes.

1476 Abbreviations: A.a.=Acrocanthosaurus; A.f.=Allosaurus; C.n.=Ceratosaurus;

1477 D.t.=Daspletosaurus; D.w.=Dilophosaurus; E.o.=Eustreptospondylus oxoniensis;

1478 G.c.=Giganotosaurus; G.l.=Gorgosaurus; S.h.=Sinraptor; T.b.=Tarbosaurus;

1479 T.r. $=$ Tyrannosaurus; Y.s. =Yangchuanosaurus. 
1481


1482

1483

1484 Figure 4. Phylogenetically generalized least squares regressions of (A) Agility $_{\text {force }}$ and (B)

1485 Agility $_{\text {moment }}$ for non-tyrannosaurid theropods (red), adult tyrannosaurids (dark blue), and putative

1486 juvenile tyrannosaurids (light blue), turning the body with both legs planted. Tyrannosaurids lie

1487 above or on the upper 95\% confidence limit of the regression, indicating definitively greater

1488 agility than expected for theropods overall when pivoting the body alone. See Figure , and

1489 Supplementary Information figure and R script, for data point labels. 

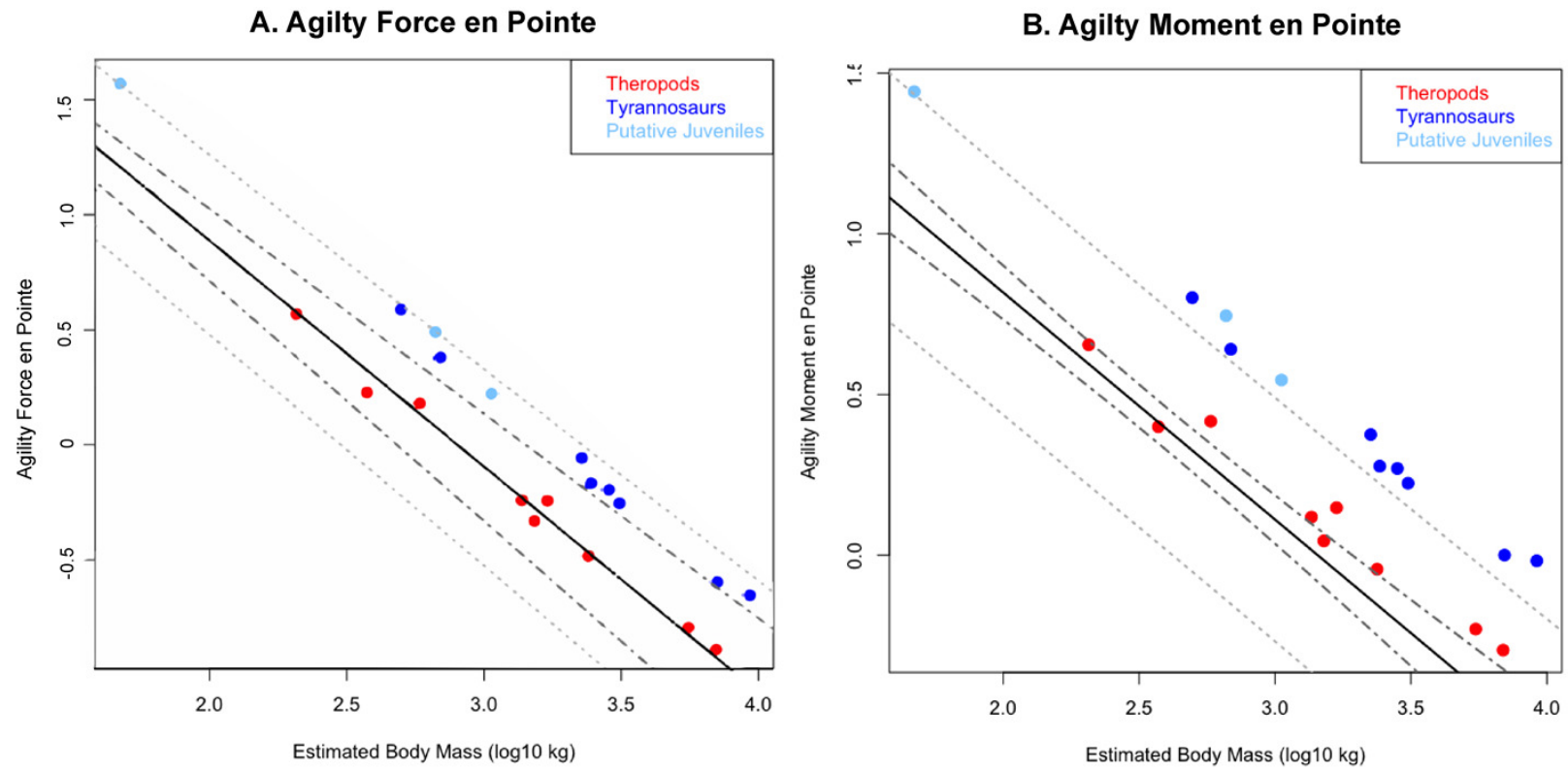

1496

1497 Figure 5. Phylogenetically generalized least squares regression of (A) Agility force and (B)

1498 Agility $_{\text {moment }}$ for non-tyrannosaurid theropods (red), adult tyrannosaurids (dark blue), and putative

1499 juvenile tyrannosaurids (light blue), when pivoting on one leg (en pointe). Tyrannosaurids lie

1500 above or on the upper 95\% confidence limit of the regression, indicating definitively greater

1501 agility than expected for theropods when pursuing prey. See Figure 1, and the Supplementary

1502 Information figure and R script, for data point labels. 
Figure 6.

1506



Figure 6. Axial body models (constructed in FreeCAD) of (A) Yangchuanosaurus shangyouensis (CV 00215), (B) Sinraptor hepingensis (ZDM 0024) and (C) Tarbosaurus bataar (ZPAL MgD-

$1514 \mathrm{I} / 4$ ) are within $0.5 \%$ of the volumes calculated by summing frusta volumes from equation 2.

1515 Three workers built different respective models, and congruence of results suggests low operator

1516 variation and high precision between the methods. The Tarbosaurus is lofted from fewer

1517 elliptical cross sections than the others, giving it a smoother appearance that nevertheless

1518 converges on the frustum results from many more cross-sections. Note that this is an exercise in

1519 cross-validation of volume estimates using uniform densities. Our mass property comparisons

1520 use frustum-based calculations that incorporate different densities for different regions of the 1521 body. 